\title{
Cortical Thickness and Surface Area Abnormalities in Bipolar I and II Disorders
}

\author{
Yoonmi Woo', Wooyoung Kang', Youbin Kang' ${ }^{2}$, Aram Kim², \\ Kyu-Man Han ${ }^{1}$, Woo-Suk Tae ${ }^{3}$, and Byung-Joo Ham ${ }^{1 凶}$ \\ ${ }^{1}$ Department of Psychiatry, Korea University Anam Hospital, Korea University College of Medicine, Seoul, Republic of Korea \\ ${ }^{2}$ Department of Biomedical Sciences, Korea University College of Medicine, Seoul, Republic of Korea \\ ${ }^{3}$ Brain Convergence Research Center, Korea University Anam Hospital, Seoul, Republic of Korea
}

Objective Although bipolar II disorder (BD II) is not simply a mitigated form of bipolar I disorder (BD I), their neurobiological differences have not been elucidated. The present study aimed to explore cortical thickness (CT) and surface area (SA) in patients with BD I and BD II and healthy controls (HCs) to investigate the shared and unique neurobiological mechanisms of BD subtypes.

Methods We enrolled 30 and 44 patients with BD I and BD II, respectively, and 100 HCs. We evaluated CT and SA using FreeSurfer and estimated differences in CT and SA among the three groups (BD I vs. BD II vs. HC). We adjusted for age, sex, educational level, and intracranial volume as confounding factors.

Results We found widespread cortical thinning in the bilateral frontal, temporal, and occipital regions; cingulate gyrus; and insula in patients with BD. Alterations in SA, including increased SA of the pars triangularis and decreased SA of the insula, were noted in patients with BD. Overall, we found BD II patients demonstrated decreased SA in the right long insula compared to BD I patients.

Conclusion Our results suggest that decreased SA in the right long insula is crucial for differentiating BD subtypes.

Psychiatry Investig 2021;18(9):850-863

Keywords Cortical thickness; Surface area; Bipolar disorder; Bipolar subtype; Long insula.

\section{INTRODUCTION}

Bipolar disorder (BD) is a recurrent and debilitating mental illness characterized by the presence of manic, hypomanic, and depressive episodes. ${ }^{1}$ More than $1 \%$ of the global population is affected by $\mathrm{BD}$, regardless of region, race, and socioeconomic status. ${ }^{2}$ Due to cognitive and functional impairments, $\mathrm{BD}$ is one of the primary causes of disability among young people (i.e., working population aged 20-50 years), leading to individual and social disease burdens. ${ }^{2,3}$ Two subtypes of $\mathrm{BD}$, type I (BD I) and II (BD II), differ in that BD I involves a full-blown manic episode, while BD II is characterized by major depres-

Received: February 24, 2021 Revised: May 21, 2021

Accepted: July 11, 2021

$\triangle$ Correspondence: Byung-Joo Ham, MD, PhD

Department of Psychiatry, Korea University Anam Hospital, Korea University College of Medicine, 73 Inchon-ro, Seongbuk-gu, Seoul 02841, Republic of Korea

Tel: +82-2-920-6843, Fax: +82-2-927-2836, E-mail: hambj@korea.ac.kr

(c) This is an Open Access article distributed under the terms of the Creative Commons Attribution Non-Commercial License (https://creativecommons.org/licenses/by$\mathrm{nc} / 4.0$ ) which permits unrestricted non-commercial use, distribution, and reproduction in any medium, provided the original work is properly cited. sive disorder combined with hypomanic episodes. ${ }^{1}$ Mood disturbances in manic episodes seen in BD I patients are severe and cause marked impairments in social functioning; however, studies have indicated that the clinical course of BD II is characterized by more recurrences, chronicity, short remission periods, higher suicide rates, and lower quality of life. ${ }^{4-7}$ This shows that $\mathrm{BD}$ II is not simply a mitigated form of BD I, suggesting that it is necessary to explore $\mathrm{BD}$ subtypes as different disease entities in neuroimaging studies. ${ }^{8}$ Unfortunately, the neurobiological differences between $\mathrm{BD}$ subtypes have not been thoroughly elucidated, as previous studies have focused on patients with BD I or have not distinguished between subtypes. ${ }^{7.8}$

Previous studies focused primarily on BD I, and many have mainly investigated cortical volume using voxel-based morphometry ${ }^{8}$ or cortical thickness (CT) using surface-based morphometry. Gray matter volume is a function of two distinct morphometries: CT and surface area (SA)., While CT reflects the number of neurons within each column, SA is reflective of the number of columns and the overall size of the cortex..$^{10,11}$ As these two parameters are the products of distinct, well-differ- 
entiated ontogenic processes, CT and SA can be separately affected by genetic or extrinsic factors. ${ }^{12,13}$ In addition, SA may be determined earlier in the neurodevelopmental period, such as during embryonic and neonatal life, and is less affected by environmental factors. ${ }^{12,14}$ Therefore, in terms of brain endophenotype, investigating CT and SA together with gray matter volume enhances the sensitivity for detecting subtype differences using cortical morphometry and provides more information underpinning the pathophysiology of $\mathrm{BD}^{8,9}$

Previous studies have shown relatively consistent results regarding CT in patients with $\mathrm{BD}$. A meta-analysis found widespread cortical thinning in the anterior cingulate cortex, orbitofrontal cortex $(\mathrm{OFC})$, paracingulate, superior temporal gyrus, and prefrontal regions in patients with $\mathrm{BD}$ compared to that in healthy controls (HCs) ${ }^{10}$ Large studies, such as the ENIGMA consortium, have worked to elucidate cortical abnormalities in patients with $\mathrm{BD}$ and reported substantially decreased CT in the bilateral frontal, temporal, and parietal regions, including the ventrolateral prefrontal cortex (VLPFC), which has long been implicated in $\mathrm{BD}$ neurobiology ${ }^{11,15}$ Conversely, there are some inconsistent results regarding SA in BD patients. Some studies, ${ }^{11,16-20}$ including the ENIGMA consortium, have failed to detect significantly altered SA, but others have found enlarged SA in the superior frontal region; temporal pole $;^{13}$ postcentral, precuneus, supramarginal, and superior temporal gyrus; insula, ${ }^{21}$ and pars triangularis ${ }^{22}$ and decreased SA in the frontotemporal cortices. ${ }^{8}$

As described, there are few studies comparing BD subtypes that have explored both CT and SA. Regarding differences in CT between BD subtypes, previous research has not detected significant differences between BD subtypes; $;^{11,23,24}$ however, some studies have found cortical thinning in the right medial orbitofrontal, left superior temporal, ${ }^{25}$ and right temporal cortices ${ }^{8}$ in patients with BD I compared to that in patients with BD II. Moreover, differences in cortical SA between BD subtypes have been explored, but there have been no significant regions showing differences between BD I and II. ${ }^{8,11}$

Therefore, we aimed to explore CT and SA in patients with $\mathrm{BD}$ I and BD II and HCs to investigate shared and unique neurobiological mechanisms. We hypothesized that patients with BD I and II would show common and distinct cortical abnormalities. We expected to identify a differential marker to compare BD subtypes. Finally, we aimed to better understand the associations between symptom severity and illness duration based on CT and SA.

\section{METHODS}

\section{Participants}

Between January 2015 and March 2019, 30 and 44 patients diagnosed with BD I and BD II, respectively, were recruited from outpatient clinics in Korea University Anam Hospital in Seoul, South Korea. Board-certified trained psychiatrists (BJ Ham and KM Han) confirmed the diagnosis of BD I and BD II using the Structured Clinical Interview for DSM-IV Axis I disorders (SCID-I) based on the Diagnostic and Statistical Manual of Mental Disorders, 4th edition (DSM-IV). The inclusion criteria for patients with $\mathrm{BD}$ were as follows: 1) age 19-64 years and 2) current euthymic or depressive state. Exclusion criteria for the patient and HC groups were as follows: 1) major psychiatric disorders other than $\mathrm{BD}$ (based on the DSM-IV criteria), including substance use or personality disorders, as comorbidities; 2) serious medical diseases or primary neurologic illnesses such as renal failure, cerebrovascular disease, organic brain damage, and epilepsy; 3) comorbid personality disorder; 4) comorbid alcohol or substance addiction (based on the DSM-IV criteria); 5) pregnancy; and 6) any contraindication for magnetic resonance imaging ${ }^{26}$ such as implanted pacemaker or claustrophobia. One hundred age- and sex-matched HCs were recruited via advertisements within the community. Boardcertified psychiatrists performed an interview to confirm that the HCs had no current or previous Axis I or Axis II disorders. We evaluated the severity of mood symptoms in $74 \mathrm{BD}$ patients using the 17-item Hamilton Depression Rating Scale (HDRS) ${ }^{27}$ and Young Mania Rating Scale (YMRS) ${ }^{28}$ Informed consent was obtained from all participants after providing a thorough explanation of the study. The present study was approved by the Institutional Review Board of Korea University Anam Hospital (IRB No: 2015AN0009) and followed the principles of the Declaration of Helsinki.

\section{MRI data acquisition and image processing}

All participants underwent $\mathrm{T}-1$ weighted imaging, performed on a 3.0 Tesla Siemens Trio whole-body imaging system (Siemens Medical Systems, Erlangen, Germany). T1-weighted images were acquired parallel to the anterior commissure-posterior commissure line using T1-weighted magnetization-prepared rapid gradient-echo (MP-RAGE) imaging (repetition time= $1,900 \mathrm{~ms}$, echo time $=2.6 \mathrm{~ms}$, field of view $=220 \mathrm{~mm}, 256 \times$ 256 matrix size, 176 coronal slices without gap, $1 \times 1 \times 1 \mathrm{~mm}^{3}$ voxels, flip angle $=16^{\circ}$, and number of excitations $=1$ ).

We used FreeSurfer (5.3 development version; Laboratory for Computational Neuroimaging, Athinoula A. Martinos Center for Biomedical Imaging, Charlestown, MA, USA; http:// surfer.nmr.mgh.harvard.edu) to create a 3D model of cortical surface reconstructions computed from T-1 images and to analyze CT and SA. Previous publications have described in detail the technical aspects of this pipeline. ${ }^{29-35}$ Briefly, this process includes the motion correction of volumetric T1-weighted images, removal of non-brain tissue using a hybrid watershed/ 
surface deformation procedure, automated Talairach transformation of each subject's native brain, segmentation of the gray matter-white matter volumetric structures, ${ }^{32}$ inflation of the cortical surface to an average spherical surface to locate both the pial surface and the gray matter-white matter boundary, intensity normalization, and automated topology correction. ${ }^{33,34,36}$ The transition of gray/white matter and the pial boundary were detected by finding a marked change in intensity through surface deformation. Automatic parcellation per hemisphere into 74 cortical gyri and sulci and the calculation of CT and SA were performed using the FreeSurfer package according to the Des- trieux atlas. ${ }^{37}$ In our current analysis, we extracted the values of gray matter thickness and SA of 76 cortical gyri from bilateral hemispheres.

\section{Statistical analysis}

In our primary analysis, we used one-way analysis of covariance to estimate differences in CT and SA. Group variables (BD I vs. BD II vs. HC) were included as independent variables, with the extracted gray matter thickness or SA values of the 76 bilateral cortical gyri as dependent variables. We included age, sex, educational level, and total intracranial volume

Table 1. Demographic and clinical characteristics of patients with BD and HCs

\begin{tabular}{|c|c|c|c|c|}
\hline Characteristics & $\mathrm{BD} I(\mathrm{~N}=30)$ & BD II $(\mathrm{N}=44)$ & $\mathrm{HC}(\mathrm{N}=100)$ & $\mathrm{p}$-value $\left(\mathrm{F}, \mathrm{t}, \chi^{2}\right)$ \\
\hline Age (yr) & $38.03 \pm 11.46$ & $32.41 \pm 9.80$ & $35.09 \pm 11.58$ & $0.103(\mathrm{~F}=2.302)$ \\
\hline Sex (male/female) & $9 / 21$ & $15 / 29$ & $37 / 63$ & $0.771\left(\chi^{2}=0.521\right)$ \\
\hline Education level & & & & $0.364\left(\chi^{2}=4.324\right)$ \\
\hline Elementary and middle school & 1 & 1 & 5 & \\
\hline High school or college/university & 26 & 42 & 83 & \\
\hline Above graduate school & 3 & 1 & 12 & \\
\hline Total intracranial volume & $1410 \pm 101$ & $1416 \pm 129$ & $1465 \pm 151$ & $0.057(\mathrm{~F}=2.909)$ \\
\hline HDRS & $4.77 \pm 4.07$ & $8.36 \pm 5.48$ & $1.78 \pm 1.92$ & $6.88 \times 10^{-19}(\mathrm{~F}=53.940)$ \\
\hline YMRS & $1.97 \pm 2.46$ & $0.75 \pm 1.24$ & NA & $0.017(\mathrm{t}=2.504)$ \\
\hline K-MDQ & $8.13 \pm 4.22$ & $9.32 \pm 2.87$ & NA & $0.186(\mathrm{t}=-1.342)$ \\
\hline HAS & $5.87 \pm 4.06$ & $7.80 \pm 6.36$ & NA & $0.146(\mathrm{t}=-1.468)$ \\
\hline BIS & $50.73 \pm 8.63$ & $55.20 \pm 10.88$ & NA & $0.064(\mathrm{t}=-1.883)$ \\
\hline Family history of mood disorder (N) & 10 & 18 & NA & $0.509\left(\chi^{2}=0.435\right)$ \\
\hline Euthymic (or remission) state/depressive state & $23 / 7$ & $23 / 21$ & NA & $0.034\left(\chi^{2}=4.513\right)$ \\
\hline Duration of illness (months) & $97.83 \pm 95.10$ & $50.09 \pm 72.60$ & NA & $0.024(\mathrm{t}=2.326)$ \\
\hline Drug-treated patients $(\mathrm{N})$ & 30 & 43 & NA & $0.406\left(\chi^{2}=0.691\right)$ \\
\hline \multicolumn{5}{|l|}{ Medication (N) } \\
\hline SSRI & 3 & 10 & & \\
\hline SNRI & 1 & 3 & & \\
\hline NDRI & 2 & 8 & & \\
\hline NaSSA & 0 & 2 & & \\
\hline Combination of $\mathrm{AD}$ & 0 & 2 & & \\
\hline Lithium & 8 & 4 & & \\
\hline AED & 18 & 37 & & \\
\hline Lithium+AED & 4 & 0 & & \\
\hline $\mathrm{AED}+\mathrm{AED}$ & 1 & 0 & & \\
\hline $\mathrm{AP}$ & 28 & 27 & & \\
\hline Combination of AP & 14 & 4 & & \\
\hline
\end{tabular}

The $\mathrm{p}$ value for comparison in age, TICV, and HDRS scores were obtained using the ANOVA test. The $\mathrm{p}$ value for sex and educational level distribution was obtained by chi-squared test. The $\mathrm{p}$ value for comparison in YMRS, K-MDQ, BIS, HAS scores were obtained using student's t-test. BD, bipolar disorder; HC, healthy control; BD I, bipolar I disorder; BD II, bipolar II disorder; HDRS, Hamilton Depression Rating Scale; YMRS, Young Mania Rating Scale; K-MDQ, Korean version of Mood Disorder Questionnaire; HAS, Hamilton Anxiety Rating Scale; BIS, Barratt Impulsiveness Scale; SSRI, selective serotonin reuptake inhibitor; NDRI, norepinephrine-dopamine reuptake inhibitor; NaSSA, noradrenergic and specific serotonergic antidepressant; Combination of Ads, combination of two or more antidepressants; AED, anti-epileptic drugs; AP, antipsychotics; AD, antidepressants 
Y Woo et al.

Table 2. Comparison of cortical thickness between patients with BD I and BD II and HCs

\begin{tabular}{|c|c|c|c|c|c|}
\hline \multirow{2}{*}{ Cortical regions } & \multirow{2}{*}{$\frac{\mathrm{BD} I(\mathrm{~N}=30)}{\text { Mean } \pm \mathrm{SD}}$} & \multirow{2}{*}{$\begin{array}{c}\text { BD II }(\mathrm{N}=44) \\
\text { Mean } \pm \text { SD }\end{array}$} & \multirow{2}{*}{$\frac{\mathrm{HC}(\mathrm{N}=100)}{\text { Mean } \pm \mathrm{SD}}$} & \multicolumn{2}{|c|}{ All groups } \\
\hline & & & & F & $\mathrm{p}$ \\
\hline \multicolumn{6}{|l|}{ Left hemisphere } \\
\hline L Frontomarginal gyrus & $2.264 \pm 0.028$ & $2.265 \pm 0.023$ & $2.403 \pm 0.015$ & 16.903 & $2.07 \times 10^{-7}$ \\
\hline L Inferior occipital gyrus & $2.325 \pm 0.036$ & $2.407 \pm 0.030$ & $2.529 \pm 0.020$ & 14.553 & $1.49 \times 10^{-6}$ \\
\hline L Subcentral gyrus & $2.606 \pm 0.035$ & $2.620 \pm 0.029$ & $2.764 \pm 0.019$ & 12.947 & $5.92 \times 10^{-6}$ \\
\hline L Transverse frontopolar gyrus & $2.543 \pm 0.038$ & $2.612 \pm 0.032$ & $2.705 \pm 0.021$ & 7.824 & $5.65 \times 10^{-4}$ \\
\hline L Anterior cingulate gyrus & $2.687 \pm 0.024$ & $2.657 \pm 0.020$ & $2.851 \pm 0.013$ & 39.210 & $1.09 \times 10^{-14}$ \\
\hline L Anterior mid-cingulate gyrus & $2.602 \pm 0.031$ & $2.576 \pm 0.026$ & $2.744 \pm 0.017$ & 18.195 & $7.10 \times 10^{-8}$ \\
\hline L Dorsal posterior cingulate gyrus & $2.611 \pm 0.052$ & $2.562 \pm 0.043$ & $2.911 \pm 0.028$ & 28.064 & $3.11 \times 10^{-11}$ \\
\hline L Ventral posterior cingulate gyrus & $2.235 \pm 0.069$ & $2.157 \pm 0.058$ & $2.554 \pm 0.038$ & 19.600 & $2.26 \times 10^{-8}$ \\
\hline L Cuneus & $1.947 \pm 0.059$ & $1.984 \pm 0.049$ & $1.763 \pm 0.033$ & 8.344 & $3.52 \times 10^{-4}$ \\
\hline L Pars opercularis & $2.675 \pm 0.033$ & $2.754 \pm 0.028$ & $2.845 \pm 0.018$ & 11.296 & $2.51 \times 10^{-5}$ \\
\hline L Pars orbitalis & $2.673 \pm 0.043$ & $2.661 \pm 0.036$ & $2.820 \pm 0.023$ & 8.977 & $1.98 \times 10^{-4}$ \\
\hline L Pars triangularis & $2.563 \pm 0.030$ & $2.624 \pm 0.025$ & $2.714 \pm 0.017$ & 11.151 & $2.85 \times 10^{-5}$ \\
\hline L Short insular gyrus & $3.189 \pm 0.073$ & $3.277 \pm 0.060$ & $3.533 \pm 0.040$ & 11.650 & $1.84 \times 10^{-5}$ \\
\hline L Middle occipital gyrus & $2.372 \pm 0.040$ & $2.406 \pm 0.033$ & $2.593 \pm 0.022$ & 18.026 & $8.16 \times 10^{-8}$ \\
\hline L Lateral occipito-temporal gyrus & $2.427 \pm 0.069$ & $2.489 \pm 0.058$ & $2.813 \pm 0.038$ & 17.980 & $8.48 \times 10^{-8}$ \\
\hline L Lingual gyrus & $2.155 \pm 0.061$ & $2.138 \pm 0.051$ & $1.905 \pm 0.034$ & 10.559 & $4.81 \times 10^{-5}$ \\
\hline L Parahippocampal gyrus & $2.787 \pm 0.048$ & $2.824 \pm 0.040$ & $3.006 \pm 0.026$ & 11.776 & $1.64 \times 10^{-5}$ \\
\hline L Angular gyrus & $2.630 \pm 0.027$ & $2.626 \pm 0.023$ & $2.736 \pm 0.015$ & 10.876 & $3.63 \times 10^{-5}$ \\
\hline L Supramarginal gyrus & $2.594 \pm 0.036$ & $2.600 \pm 0.030$ & $2.804 \pm 0.020$ & 23.257 & $1.23 \times 10^{-9}$ \\
\hline L Superior parietal lobule & $2.255 \pm 0.033$ & $2.293 \pm 0.028$ & $2.430 \pm 0.018$ & 14.924 & $1.09 \times 10^{-6}$ \\
\hline L Precentral gyrus & $2.680 \pm 0.040$ & $2.693 \pm 0.033$ & $2.880 \pm 0.022$ & 16.250 & $3.56 \times 10^{-7}$ \\
\hline L Subcallosal gyrus & $2.206 \pm 0.070$ & $2.261 \pm 0.059$ & $2.493 \pm 0.039$ & 9.247 & $1.55 \times 10^{-4}$ \\
\hline L Anterior transverse temporal gyrus & $2.362 \pm 0.050$ & $2.460 \pm 0.042$ & $2.255 \pm 0.027$ & 8.560 & $2.89 \times 10^{-4}$ \\
\hline L Planum polare & $3.036 \pm 0.064$ & $3.104 \pm 0.053$ & $3.437 \pm 0.035$ & 22.392 & $2.43 \times 10^{-9}$ \\
\hline L Middle temporal gyrus & $2.731 \pm 0.056$ & $2.758 \pm 0.046$ & $3.074 \pm 0.030$ & 24.043 & $6.66 \times 10^{-10}$ \\
\hline \multicolumn{6}{|l|}{ Right hemisphere } \\
\hline R Frontomarginal gyrus & $2.284 \pm 0.034$ & $2.296 \pm 0.028$ & $2.421 \pm 0.018$ & 10.278 & $6.17 \times 10^{-5}$ \\
\hline R Inferior occipital gyrus & $2.505 \pm 0.046$ & $2.485 \pm 0.038$ & $2.695 \pm 0.025$ & 13.434 & $3.89 \times 10^{-6}$ \\
\hline R Subcentral gyrus & $2.590 \pm 0.036$ & $2.574 \pm 0.030$ & $2.785 \pm 0.019$ & 22.776 & $1.79 \times 10^{-9}$ \\
\hline R Transverse frontopolar gyrus & $2.477 \pm 0.033$ & $2.507 \pm 0.027$ & $2.675 \pm 0.018$ & 21.157 & $6.46 \times 10^{-9}$ \\
\hline R Anterior cingulate gyrus & $2.682 \pm 0.027$ & $2.627 \pm 0.023$ & $2.825 \pm 0.015$ & 29.606 & $9.88 \times 10^{-12}$ \\
\hline R Anterior mid-cingulate gyrus & $2.671 \pm 0.026$ & $2.626 \pm 0.022$ & $2.830 \pm 0.014$ & 35.687 & $1.25 \times 10^{-13}$ \\
\hline R Dorsal posterior cingulate gyrus & $2.770 \pm 0.039$ & $2.739 \pm 0.032$ & $2.952 \pm 0.021$ & 18.680 & $4.77 \times 10^{-8}$ \\
\hline R Ventral posterior cingulate gyrus & $2.348 \pm 0.073$ & $2.366 \pm 0.061$ & $2.653 \pm 0.040$ & 11.337 & $2.42 \times 10^{-5}$ \\
\hline R Cuneus & $1.945 \pm 0.061$ & $2.028 \pm 0.051$ & $1.792 \pm 0.033$ & 8.203 & $4.00 \times 10^{-4}$ \\
\hline R Pars opercularis & $2.661 \pm 0.030$ & $2.735 \pm 0.025$ & $2.881 \pm 0.017$ & 25.036 & $3.09 \times 10^{-10}$ \\
\hline R Pars orbitalis & $2.667 \pm 0.041$ & $2.747 \pm 0.034$ & $2.844 \pm 0.023$ & 7.898 & $5.28 \times 10^{-4}$ \\
\hline $\mathrm{R}$ Pars triangularis & $2.540 \pm 0.029$ & $2.656 \pm 0.024$ & $2.756 \pm 0.016$ & 22.921 & $1.60 \times 10^{-9}$ \\
\hline R Middle frontal gyrus & $2.653 \pm 0.028$ & $2.665 \pm 0.024$ & $2.767 \pm 0.016$ & 9.946 & $8.30 \times 10^{-5}$ \\
\hline R Middle occipital gyrus & $2.467 \pm 0.039$ & $2.436 \pm 0.033$ & $2.626 \pm 0.022$ & 14.116 & $2.17 \times 10^{-6}$ \\
\hline R Lateral occipito-temporal gyrus & $2.454 \pm 0.062$ & $2.517 \pm 0.051$ & $2.806 \pm 0.034$ & 18.349 & $6.26 \times 10^{-8}$ \\
\hline R Lingual gyrus & $2.187 \pm 0.055$ & $2.201 \pm 0.046$ & $1.957 \pm 0.030$ & 12.929 & $6.02 \times 10^{-6}$ \\
\hline
\end{tabular}


Table 2. Comparison of cortical thickness between patients with BD I and BD II and HCs (continued)

\begin{tabular}{|c|c|c|c|c|c|}
\hline \multirow{2}{*}{ Cortical regions } & \multirow{2}{*}{$\begin{array}{c}\text { BD I }(\mathrm{N}=30) \\
\text { Mean } \pm \text { SD }\end{array}$} & \multirow{2}{*}{$\begin{array}{c}\text { BD II }(\mathrm{N}=44) \\
\text { Mean } \pm \text { SD }\end{array}$} & \multirow{2}{*}{$\begin{array}{c}\mathrm{HC}(\mathrm{N}=100) \\
\text { Mean } \pm \mathrm{SD}\end{array}$} & \multicolumn{2}{|c|}{ All groups } \\
\hline & & & & $\mathrm{F}$ & $\mathrm{p}$ \\
\hline R Parahippocampal gyrus & $2.889 \pm 0.054$ & $2.885 \pm 0.045$ & $3.073 \pm 0.030$ & 8.241 & $3.86 \times 10^{-4}$ \\
\hline R Orbital gyrus & $2.610 \pm 0.032$ & $2.687 \pm 0.026$ & $2.774 \pm 0.017$ & 11.481 & $2.13 \times 10^{-5}$ \\
\hline R Angular gyrus & $2.573 \pm 0.026$ & $2.609 \pm 0.022$ & $2.716 \pm 0.014$ & 15.359 & $7.53 \times 10^{-7}$ \\
\hline R Supramarginal gyrus & $2.576 \pm 0.036$ & $2.602 \pm 0.030$ & $2.811 \pm 0.020$ & 25.634 & $1.96 \times 10^{-10}$ \\
\hline R Superior parietal lobule & $2.260 \pm 0.033$ & $2.284 \pm 0.028$ & $2.411 \pm 0.018$ & 11.888 & $1.49 \times 10^{-5}$ \\
\hline R Precentral gyrus & $2.664 \pm 0.039$ & $2.701 \pm 0.032$ & $2.838 \pm 0.021$ & 10.919 & $3.49 \times 10^{-5}$ \\
\hline R Planum polare & $2.974 \pm 0.063$ & $3.016 \pm 0.052$ & $3.222 \pm 0.034$ & 8.890 & $2.14 \times 10^{-4}$ \\
\hline R Middle temporal gyrus & $2.759 \pm 0.051$ & $2.779 \pm 0.043$ & $3.059 \pm 0.028$ & 21.714 & $4.15 \times 10^{-9}$ \\
\hline
\end{tabular}

The general linear model (GLM) was adjusted for age, sex, educational level, and intracranial volume as covariates. Regions that remained significant after Bonferroni correction are listed: 76 regions of cortex, corrected $\mathrm{p}=(0.05 / 76)=6.58 \times 10^{-4}$. BD I, bipolar disorder type I; BD II, bipolar disorder type II; HC, healthy controls; SD, standard deviation

(TICV) as covariates to prevent potential confounding effects. For cortical regions indicated to have significant alterations in our main analysis, post-hoc analyses were performed to determine significant pairwise comparisons after Bonferroni tests. For multiple comparisons, Bonferroni corrections were applied to minimize the type I error risk: $\mathrm{p}<0.05 / 76$ (number of comparisons in bilateral hemispheres $)=6.58 \times 10^{-4}$.

In an additional analysis, we performed a two-tailed Pearson's partial correlation test to examine the correlation of illness duration and HDRS scores with CT or SA in the BD groups. Cortical regions with significant differences among the three groups in the main analyses were included in the additional analysis. Correlation analysis was performed with age, sex, educational level, and TICV as covariates.

To analyze group differences in demographic and clinical variables, we performed analysis of variance ${ }^{38}$ for continuous variables (i.e., age, TICV, and HDRS scores) and chi-squared tests for categorical variables (i.e., sex, educational level, family history, mood state, and drug treatment). We also performed t-tests to compare illness duration and psychiatric scale scores, including the YMRS, Korean version of the Mood Disorder Questionnaire, Hamilton Anxiety Rating Scale, and Barratt Impulsiveness Scale scores, between the BD I and BD II groups. All statistical analyses were performed using SPSS (version 24.0; IBM Corp., Armonk, NY, USA).

\section{RESULTS}

\section{Demographic and clinical characteristics}

The demographic and clinical variables of 30 patients with BD I, 44 patients with BD II, and $100 \mathrm{HCs}$, including age, sex, educational levels, TICV, mood state, and psychotropic medications, are presented in Table 1. No significant differences were found in age, sex, educational level, and TICV. There were significant differences in the HDRS score among the BD I, BD
II, and HC groups $(\mathrm{F}=53.940, \mathrm{p}<0.001)$. For the mood state, seven patients with $\mathrm{BD}$ I and 21 patients with $\mathrm{BD}$ II were in a depressive episode, while the rest of the patients were in a euthymic state $(\mathrm{p}<0.05)$. Although the YMRS scores in the BD I group were significantly higher than those in the $\mathrm{BD}$ II group $(\mathrm{p}<0.05)$, none of the patients with BD I or BD II showed a minimum score for manic symptom severity of 13 points. ${ }^{28,39}$ The illness duration in the BD I group was significantly longer than that in the BD II group. All patients with BD I and 43 patients with $\mathrm{BD}$ II were taking psychotropic medications at the time of enrollment. Detailed information about psychotropic medications used by $\mathrm{BD}$ patients is described in Table 1 .

\section{CT analysis}

\section{Cortical thinning in patients with $\mathrm{BD}$ compared to that in $\mathrm{HCs}$}

We detected significant and widespread cortical thinning in diverse regions along the bilateral frontal, temporal, parietal, and occipital regions; cingulate gyrus; and left insula in both patients with BD I and BD II. Forty-nine cortical regions of the 76 cortical gyri demonstrated significant differences, and the results of CT analysis are presented in Tables 2 and 3. Notably, the regions with the largest differences on comparison between patients with BD I and BD II and HCs involved the bilateral cingulate gyrus, including the left anterior cingulate gyrus $(\mathrm{p}=$ $\left.1.09 \times 10^{-14}\right)$, right anterior mid-cingulate gyrus $\left(\mathrm{p}=1.25 \times 10^{-13}\right)$, and right anterior cingulate gyrus $\left(\mathrm{p}=9.88 \times 10^{-12}\right)$. Patients with BD I showed more widespread cortical thinning than patients with BD II and HCs. Some areas of the VLPFC (including the pars triangularis, left pars opercularis, and right pars orbitalis of the inferior frontal gyrus) and the OFC (including the right orbital gyrus) showed significant cortical thinning only in patients with BD I but not in patients with BD II, compared to that in HCs; however, the left pars orbitalis showed significant 
Table 3. Post-hoc comparison of cortical thickness between patients with BD I and BD II and HCs

\begin{tabular}{|c|c|c|c|c|}
\hline \multirow{2}{*}{ Cortical regions } & BD I vs. HC & BD II vs. HC & BD I vs. BD II & \multirow{2}{*}{ Comparison } \\
\hline & $\mathrm{p}$ & $\mathrm{p}$ & $\mathrm{p}$ & \\
\hline \multicolumn{5}{|l|}{ Left hemisphere } \\
\hline L Frontomarginal gyrus & $2.38 \times 10^{-5}$ & $2.36 \times 10^{-6}$ & 0.971 & $\mathrm{BD}$ I \& BD II $<\mathrm{HC}$ \\
\hline L Inferior occipital gyrus & $1.73 \times 10^{-6}$ & $8.94 \times 10^{-4}$ & 0.085 & $\mathrm{BD} \mathrm{I}<\mathrm{HC}$ \\
\hline L Subcentral gyrus & $1.01 \times 10^{-4}$ & $5.90 \times 10^{-5}$ & 0.758 & $\mathrm{BD}$ I \& $\mathrm{BD}$ II $<\mathrm{HC}$ \\
\hline L Transverse frontopolar gyrus & $3.14 \times 10^{-4}$ & 0.018 & 0.169 & $\mathrm{BD} \mathrm{I}<\mathrm{HC}$ \\
\hline L Anterior cingulate gyrus & $1.84 \times 10^{-8}$ & $2.79 \times 10^{-13}$ & 0.344 & $\mathrm{BD} \mathrm{I} \& \mathrm{BD}$ II $<\mathrm{HC}$ \\
\hline L Anterior mid-cingulate gyrus & $8.79 \times 10^{-5}$ & $2.11 \times 10^{-7}$ & 0.512 & $\mathrm{BD}$ I \& $\mathrm{BD}$ II $<\mathrm{HC}$ \\
\hline L Dorsal posterior cingulate gyrus & $1.06 \times 10^{-6}$ & $3.39 \times 10^{-10}$ & 0.474 & $\mathrm{BD}$ I \& $\mathrm{BD}$ II $<\mathrm{HC}$ \\
\hline L Ventral posterior cingulate gyrus & $8.33 \times 10^{-5}$ & $8.33 \times 10^{-5}$ & 0.388 & $\mathrm{BD}$ I \& $\mathrm{BD}$ II $<\mathrm{HC}$ \\
\hline L Cuneus & 0.008 & $3.13 \times 10^{-4}$ & 0.633 & $\mathrm{BD} \mathrm{II}>\mathrm{HC}$ \\
\hline L Pars opercularis & $1.33 \times 10^{-5}$ & 0.007 & 0.067 & $\mathrm{BD} \mathrm{I}<\mathrm{HC}$ \\
\hline L Pars orbitalis & 0.003 & $3.08 \times 10^{-4}$ & 0.833 & $\mathrm{BD} \mathrm{II}<\mathrm{HC}$ \\
\hline L Pars triangularis & $2.34 \times 10^{-5}$ & 0.004 & 0.124 & $\mathrm{BD} \mathrm{I}<\mathrm{HC}$ \\
\hline L Short insular gyrus & $5.33 \times 10^{-5}$ & $6.07 \times 10^{-4}$ & 0.350 & $\mathrm{BD}$ I \& $\mathrm{BD}$ II $<\mathrm{HC}$ \\
\hline L Middle occipital gyrus & $2.45 \times 10^{-6}$ & $5.82 \times 10^{-6}$ & 0.509 & $\mathrm{BD}$ I \& $\mathrm{BD}$ II $<\mathrm{HC}$ \\
\hline L Lateral occipito-temporal gyrus & $2.31 \times 10^{-6}$ & $6.55 \times 10^{-6}$ & 0.488 & $\mathrm{BD}$ I \& BD II $<\mathrm{HC}$ \\
\hline L Lingual gyrus & $4.97 \times 10^{-4}$ & $2.3 \times 10^{-4}$ & 0.837 & $\mathrm{BD}$ I \& $\mathrm{BD}$ II $>\mathrm{HC}$ \\
\hline L Parahippocampal gyrus & $1.05 \times 10^{-4}$ & $2.4 \times 10^{-4}$ & 0.560 & $\mathrm{BD}$ I \& BD II $<\mathrm{HC}$ \\
\hline L Angular gyrus & $8.2 \times 10^{-4}$ & $1.01 \times 10^{-4}$ & 0.928 & $\mathrm{BD}$ II $<\mathrm{HC}$ \\
\hline L Supramarginal gyrus & $6.74 \times 10^{-7}$ & $5.85 \times 10^{-8}$ & 0.887 & $\mathrm{BD}$ I \& $\mathrm{BD}$ II $<\mathrm{HC}$ \\
\hline L Superior parietal lobule & $8.43 \times 10^{-6}$ & $6.9 \times 10^{-5}$ & 0.382 & $\mathrm{BD}$ I $\&$ BD II $<\mathrm{HC}$ \\
\hline L Precentral gyrus & $1.87 \times 10^{-5}$ & $6.15 \times 10^{-6}$ & 0.798 & $\mathrm{BD}$ I $\&$ BD II $<\mathrm{HC}$ \\
\hline L Subcallosal gyrus & $4.71 \times 10^{-4}$ & $1.29 \times 10^{-3}$ & 0.551 & $\mathrm{BD} \mathrm{I}<\mathrm{HC}$ \\
\hline L Anterior transverse temporal gyrus & 0.063 & $7.58 \times 10^{-5}$ & 0.137 & $\mathrm{BD}$ II $>\mathrm{HC}$ \\
\hline L Planum polare & $1.52 \times 10^{-7}$ & $6.56 \times 10^{-7}$ & 0.418 & $\mathrm{BD}$ I \& $\mathrm{BD}$ II $<\mathrm{HC}$ \\
\hline L Middle temporal gyrus & $2.32 \times 10^{-7}$ & $6.79 \times 10^{-8}$ & 0.715 & $\mathrm{BD}$ I \& $\mathrm{BD}$ II $<\mathrm{HC}$ \\
\hline \multicolumn{5}{|l|}{ Right hemisphere } \\
\hline R Frontomarginal gyrus & $5.02 \times 10^{-4}$ & $3.22 \times 10^{-4}$ & 0.784 & $\mathrm{BD}$ I $\&$ BD II $<\mathrm{HC}$ \\
\hline R Inferior occipital gyrus & $3.87 \times 10^{-4}$ & $1.04 \times 10^{-5}$ & 0.737 & $\mathrm{BD}$ I \& $\mathrm{BD}$ II $<\mathrm{HC}$ \\
\hline R Subcentral gyrus & $3.69 \times 10^{-6}$ & $2.27 \times 10^{-8}$ & 0.737 & $\mathrm{BD}$ I \& BD II $<\mathrm{HC}$ \\
\hline R Transverse frontopolar gyrus & $4.15 \times 10^{-7}$ & $9.49 \times 10^{-7}$ & 0.492 & $\mathrm{BD}$ I \& $\mathrm{BD}$ II $<\mathrm{HC}$ \\
\hline R Anterior cingulate gyrus & $9.39 \times 10^{-6}$ & $2.03 \times 10^{-11}$ & 0.124 & BD I \& BD II $<\mathrm{HC}$ \\
\hline R Anterior mid-cingulate gyrus & $3.34 \times 10^{-7}$ & $8.25 \times 10^{-13}$ & 0.186 & $\mathrm{BD}$ I $\&$ BD II $<\mathrm{HC}$ \\
\hline $\mathrm{R}$ Dorsal posterior cingulate gyrus & $6.24 \times 10^{-5}$ & $1.65 \times 10^{-7}$ & 0.573 & $\mathrm{BD}$ I $\&$ BD II $<\mathrm{HC}$ \\
\hline $\mathrm{R}$ Ventral posterior cingulate gyrus & $3.24 \times 10^{-4}$ & $1.34 \times 10^{-4}$ & 0.842 & $\mathrm{BD} \mathrm{I} \& \mathrm{BD}$ II $<\mathrm{HC}$ \\
\hline R Cuneus & 0.029 & $1.58 \times 10^{-4}$ & 0.295 & $\mathrm{BD}$ II $>\mathrm{HC}$ \\
\hline R Pars opercularis & $2.24 \times 10^{-9}$ & $4.15 \times 10^{-6}$ & 0.064 & BD I \& BD II $<\mathrm{HC}$ \\
\hline $\mathrm{R}$ Pars orbitalis & $2.56 \times 10^{-4}$ & 0.022 & 0.138 & $\mathrm{BD} \mathrm{I}<\mathrm{HC}$ \\
\hline $\mathrm{R}$ Pars triangularis & $7.15 \times 10^{-10}$ & $7.18 \times 10^{-4}$ & 0.002 & $\mathrm{BD} \mathrm{I}<\mathrm{HC}$ \\
\hline R Middle frontal gyrus & $5.63 \times 10^{-4}$ & $4.35 \times 10^{-4}$ & 0.756 & $\mathrm{BD}$ I $\&$ BD II $<\mathrm{HC}$ \\
\hline R Middle occipital gyrus & $5.61 \times 10^{-4}$ & $3.78 \times 10^{-6}$ & 0.543 & $\mathrm{BD} \mathrm{I} \& \mathrm{BD}$ II $<\mathrm{HC}$ \\
\hline R Lateral occipito-temporal gyrus & $1.53 \times 10^{-6}$ & $6.51 \times 10^{-6}$ & 0.440 & $\mathrm{BD}$ I \& BD II $<\mathrm{HC}$ \\
\hline R Lingual gyrus & $3.50 \times 10^{-4}$ & $1.98 \times 10^{-5}$ & 0.847 & $\mathrm{BD}$ I \& $\mathrm{BD}$ II $>\mathrm{HC}$ \\
\hline
\end{tabular}


Table 3. Post-hoc comparison of cortical thickness between patients with BD I and BD II and HCs (continued)

\begin{tabular}{|c|c|c|c|c|}
\hline \multirow{2}{*}{ Cortical regions } & BD I vs. HC & BD II vs. HC & BD I vs. BD II & \multirow{2}{*}{ Comparison } \\
\hline & $\mathrm{p}$ & $\mathrm{p}$ & $\mathrm{p}$ & \\
\hline R Orbital gyrus & $1.10 \times 10^{-5}$ & 0.007 & 0.062 & $\mathrm{BD} \mathrm{I}<\mathrm{HC}$ \\
\hline R Angular gyrus & $4.64 \times 10^{-6}$ & $7.84 \times 10^{-5}$ & 0.307 & $\mathrm{BD}$ I \& BD II $<\mathrm{HC}$ \\
\hline R Supramarginal gyrus & $5.92 \times 10^{-8}$ & $4.55 \times 10^{-8}$ & 0.582 & $\mathrm{BD}$ I \& BD II $<\mathrm{HC}$ \\
\hline R Superior parietal lobule & $1.02 \times 10^{-4}$ & $2.14 \times 10^{-4}$ & 0.572 & BD I \& $\mathrm{BD}$ II $<\mathrm{HC}$ \\
\hline R Precentral gyrus & $1.31 \times 10^{-4}$ & $5.71 \times 10^{-4}$ & 0.473 & BD I \& BD II $<\mathrm{HC}$ \\
\hline R Middle temporal gyrus & $9.34 \times 10^{-7}$ & $2.28 \times 10^{-7}$ & 0.766 & $\mathrm{BD}$ I \& BD II $<\mathrm{HC}$ \\
\hline
\end{tabular}

The general linear model (GLM) was adjusted for age, sex, educational level, and intracranial volume as covariates. Regions that remained significant after Bonferroni correction are listed: 76 regions of cortex, corrected $\mathrm{p}=(0.05 / 76)=6.58 \times 10^{-4}$. BD I, bipolar disorder type I; BD II, bipolar disorder type II; HC, healthy controls; SD, standard deviation

cortical thinning in patients with BD II but not in patients with BD I.

\section{No significant differences in CT between BD subtypes \\ In our post-hoc analysis comparing CT between BD I and II patients (Table 3), there were no significant regions showing differences between patients with BD I and BD II after correc- tion for multiple comparisons. Although BD I patients demon- strated lower $\mathrm{CT}$ than $\mathrm{BD}$ II patients in the right pars triangu- laris ( $\mathrm{p}=0.002)$, this difference did not remain significant after Bonferroni correction.}

\section{Cortical SA analysis}

\section{Mixed results for cortical SA in patients with BD I and}

\section{BD II compared to that in $\mathrm{HCs}$}

Diverse regions with significantly different SA in patients with BD compared to that in HCs are shown in Tables 4 and 5. We detected various regions with significantly decreased SA in both $\mathrm{BD}$ I and II patients compared to that in HCs, with the largest differences noted in the left long insula $\left(\mathrm{p}=1.80 \times 10^{-14}\right)$, right straight gyrus $\left(\mathrm{p}=2.18 \times 10^{-9}\right)$, left pars orbitalis $(\mathrm{p}=2.28 \times$ $\left.10^{-9}\right)$, right anterior cingulate gyrus $\left(\mathrm{p}=8.77 \times 10^{-9}\right)$, right posterior mid-cingulate gyrus $\left(\mathrm{p}=6.36 \times 10^{-7}\right)$, right lateral superior temporal gyrus $\left(\mathrm{p}=6.83 \times 10^{-7}\right)$, right subcentral gyrus $(\mathrm{p}=6.83 \times$ $\left.10^{-7}\right)$, right cuneus $\left(\mathrm{p}=1.37 \times 10^{-6}\right)$, right superior frontal gyrus $\left(\mathrm{p}=1.44 \times 10^{-5}\right)$, and right lingual gyrus $\left(\mathrm{p}=2.17 \times 10^{-5}\right)$. Cortices showing increased SA in both patients with BD I and II included the right pars triangularis $\left(\mathrm{p}=2.76 \times 10^{-6}\right)$, right ventral posterior cingulate $\left(\mathrm{p}=1.73 \times 10^{-5}\right)$, right middle frontal gyrus $\left(\mathrm{p}=2.37 \times 10^{-5}\right)$, and right anterior temporal gyrus $\left(\mathrm{p}=3.06 \times 10^{-5}\right)$.

In the present analysis, the $\mathrm{SA}$ of the right pars opercularis was significantly smaller in patients with BD II but not patients with BD I than in HCs $\left(p=3.27 \times 10^{-6}\right)$. Conversely, there were cortical areas showing a significantly increased $\mathrm{SA}$ in $\mathrm{BD}$ II patients but not in BD I patients compared to that in HCs, including the right frontomarginal gyrus $\left(\mathrm{p}=2.25 \times 10^{-5}\right)$, right parahippocampal gyrus $\left(\mathrm{p}=4.96 \times 10^{-5}\right)$, and right superior occipital gyrus $\left(\mathrm{p}=5.29 \times 10^{-5}\right)$.

\section{Right long insula as a significant region for differentiating BD subtypes}

The right long insula was shown to be a significant region for differentiating BD subtypes in the SA analysis, even after correcting for multiple comparisons. BD II patients demonstrated a significantly smaller SA in the right long insula $(\mathrm{p}=$ $6.57 \times 10^{-4}$ ) than BD I patients. In the left long insula, BD II patients showed a smaller SA than BD I patients, but this difference did not remain significant after Bonferroni correction $(\mathrm{p}=0.003)$.

\section{Association of CT and SA with illness duration and HDRS scores}

The results of the correlations of illness duration with $\mathrm{CT}$ and SA after the adjustment for age, sex, educational level, and TICV are shown in Tables 6 and 7. We found a significant positive correlation between CT in the right dorsal posterior cingulate and illness duration, but it was not significant after correction $(\mathrm{p}=0.031)$. We also explored the correlation of HDRS score with CT and SA. Negative correlations were found for $\mathrm{SA}$ in the left long insula $(\mathrm{r}=-0.250, \mathrm{p}=0.037)$ and right long insula $(r=-0.292, p=0.014)$ with HDRS scores, but these correlations did not remain significant after Bonferroni correction.

We also performed correlation analyses between illness duration or HDRS score and cortical abnormalities in patients with BD I and BD II, respectively (Supplementary Tables 1-4 in the online-only Data Supplement). In the correlation analyses, illness duration showed a positive correlation with $\mathrm{CT}$ in the left pars triangularis in $\mathrm{BD}$ II patients $(\mathrm{r}=0.361, \mathrm{p}=0.022)$ and a negative correlation with SA in the left insula in BD I patients $(\mathrm{r}=$ $-0.425, \mathrm{p}=\mathrm{p}-0.030$ ); however, these results did not persist after Bonferroni correction. Similarly, in BD I patients, HDRS score showed negative correlations with CT in the left inferior occipital gyrus $(\mathrm{r}=-0.434, \mathrm{p}=0.027)$, left dorsal posterior cingulate gy- 
Table 4. Comparison of cortical surface area between patients with BD I and BD II and HCs

\begin{tabular}{|c|c|c|c|c|c|}
\hline \multirow{2}{*}{ Cortical regions } & \multirow{2}{*}{$\begin{array}{c}\text { BD I }(\mathrm{N}=30) \\
\text { Mean } \pm \text { SD }\end{array}$} & \multirow{2}{*}{$\begin{array}{c}\text { BD II }(\mathrm{N}=44) \\
\text { Mean } \pm \text { SD }\end{array}$} & \multirow{2}{*}{$\begin{array}{c}\mathrm{HC}(\mathrm{N}=100) \\
\text { Mean } \pm \mathrm{SD}\end{array}$} & \multicolumn{2}{|c|}{ All groups } \\
\hline & & & & $\mathrm{F}$ & $\mathrm{p}$ \\
\hline \multicolumn{6}{|l|}{ Left hemisphere } \\
\hline L Pars orbitalis & $915.8 \pm 23.9$ & $898.6 \pm 19.9$ & $900.4 \pm 13.1$ & 22.469 & $2.28 \times 10^{-9}$ \\
\hline L Long insular gyrus & $425.4 \pm 15.0$ & $367.0 \pm 12.5$ & $496.3 \pm 8.2$ & 38.484 & $1.80 \times 10^{-14}$ \\
\hline \multicolumn{6}{|l|}{ Right hemisphere } \\
\hline R Frontomarginal gyrus & $705.1 \pm 25.6$ & $754.5 \pm 21.3$ & $635.1 \pm 14.0$ & 11.417 & $2.25 \times 10^{-5}$ \\
\hline R Subcentral gyrus & $899.8 \pm 24.8$ & $903.3 \pm 20.7$ & $1,017.1 \pm 13.6$ & 14.884 & $1.13 \times 10^{-6}$ \\
\hline R Transverse frontopolar gyrus & $1,068.9 \pm 64.8$ & $1,032.0 \pm 54.0$ & $814.7 \pm 35.5$ & 8.992 & $1.96 \times 10^{-4}$ \\
\hline $\mathrm{R}$ Anterior cingulate gyrus & $1,658.5 \pm 59.4$ & $1,591.3 \pm 49.5$ & $1,941.8 \pm 32.5$ & 20.774 & $8.77 \times 10^{-9}$ \\
\hline R Posterior mid-cingulate gyrus & $852.6 \pm 38.3$ & $822.0 \pm 31.9$ & $1,015.3 \pm 21.0$ & 15.560 & $6.36 \times 10^{-7}$ \\
\hline R Ventral posterior cingulate gyrus & $512.2 \pm 67.5$ & $512.5 \pm 56.2$ & $234.5 \pm 36.9$ & 11.718 & $1.73 \times 10^{-5}$ \\
\hline R Cuneus & $1,338.9 \pm 48.5$ & $1,326.5 \pm 40.4$ & $1,555.0 \pm 26.6$ & 14.654 & $1.37 \times 10^{-6}$ \\
\hline R Pars opercularis & $734.9 \pm 42.4$ & $668.8 \pm 35.3$ & $874.5 \pm 23.2$ & 12.943 & $5.94 \times 10^{-6}$ \\
\hline $\mathrm{R}$ Pars orbitalis & $421.4 \pm 35.9$ & $379.9 \pm 30.0$ & $280.0 \pm 19.7$ & 7.721 & $6.21 \times 10^{-4}$ \\
\hline $\mathrm{R}$ Pars triangularis & $1,221.0 \pm 119.2$ & $1,236.5 \pm 99.3$ & $696.8 \pm 65.2$ & 13.835 & $2.76 \times 10^{-6}$ \\
\hline R Middle frontal gyrus & $3,192.5 \pm 125.4$ & $3,180.9 \pm 104.5$ & $2,677.2 \pm 68.7$ & 11.359 & $2.37 \times 10^{-5}$ \\
\hline R Superior frontal gyrus & $3,328.3 \pm 242.8$ & $3,340.1 \pm 202.3$ & $4,344.1 \pm 132.9$ & 11.929 & $1.44 \times 10^{-5}$ \\
\hline R Long insular gyrus & $436.9 \pm 13.9$ & $374.1 \pm 11.6$ & $437.4 \pm 7.6$ & 11.022 & $3.19 \times 10^{-5}$ \\
\hline R Short insular gyrus & $771.4 \pm 69.1$ & $753.3 \pm 57.6$ & $526.7 \pm 37.8$ & 7.953 & $5.02 \times 10^{-4}$ \\
\hline R Superior occipital gyrus & $1,121.4 \pm 29.5$ & $1,202.4 \pm 24.6$ & $1,067.0 \pm 16.2$ & 10.452 & $5.29 \times 10^{-5}$ \\
\hline R Lingual gyrus & $1,694.2 \pm 74.0$ & $1,716.7 \pm 61.7$ & $2,008.5 \pm 40.5$ & 11.459 & $2.17 \times 10^{-5}$ \\
\hline R Parahippocampal gyrus & $988.8 \pm 60.8$ & $1,056.8 \pm 50.7$ & $795.4 \pm 33.3$ & 10.523 & $4.96 \times 10^{-5}$ \\
\hline R Precuneus & $1,388.7 \pm 83.4$ & $1,424.0 \pm 69.5$ & $1,730.0 \pm 45.7$ & 10.249 & $6.33 \times 10^{-5}$ \\
\hline R Straight gyrus & $444.7 \pm 22.2$ & $417.6 \pm 18.5$ & $554.5 \pm 12.2$ & 22.527 & $2.18 \times 10^{-9}$ \\
\hline $\mathrm{R}$ Anterior transverse temporal gyrus & $566.5 \pm 60.1$ & $544.2 \pm 50.1$ & $313.1 \pm 32.9$ & 11.069 & $3.06 \times 10^{-5}$ \\
\hline R Lateral superior temporal gyrus & $1,020.4 \pm 44.7$ & $1,011.9 \pm 37.3$ & $1,227.2 \pm 24.5$ & 15.476 & $6.83 \times 10^{-7}$ \\
\hline R Planum temporale & $959.4 \pm 81.3$ & $951.9 \pm 67.8$ & $650.0 \pm 44.5$ & 9.720 & $1.02 \times 10^{-4}$ \\
\hline
\end{tabular}

The general linear model (GLM) was adjusted for age, sex, educational level, and intracranial volume as covariates. Regions that remained significant after Bonferroni correction are listed: 76 regions of cortex, corrected $\mathrm{p}=(0.05 / 76)=6.58 \times 10^{-4}$. BD I, bipolar disorder type I; BD II, bipolar disorder type II; HC, healthy controls; SD, standard deviation

rus ( $\mathrm{r}=-0.439, \mathrm{p}=0.025)$, left middle occipital gyrus $(\mathrm{r}=-0.421$, $\mathrm{p}=0.032)$, and right pars orbitalis $(\mathrm{r}=-0.470, \mathrm{p}=0.015)$, and with $\mathrm{SA}$ in the right anterior cingulate gyrus $(\mathrm{r}=-0.414, \mathrm{p}=0.036)$, and right strait gyrus $(\mathrm{r}=-0.415, \mathrm{p}=0.035)$. However, the significance did not persist after controlling for multiple comparisons.

\section{Association of SA in the right long insula with YMRS score}

We performed additional correlation analyses to explore whether the decrease in SA in the right long insular gyrus was related to the lower YMRS score (Supplementary Tables 5 and 6 in the online-only Data Supplement). A positive correlation between YMRS score and SA in the right insula was found in all $\mathrm{BD}$ patients $(\mathrm{r}=0.244, \mathrm{p}=0.042)$, but this result did not remain significant when the analysis was limited to BD II patients and after a correction for multiple comparisons was performed.

\section{DISCUSSION}

In the present study, we investigated differences in CT and SA between patients with BD I and BD II. In addition to common cortical abnormalities, we also found differences specific to $\mathrm{BD}$ subtype. We aimed to explore the neurobiological mechanisms of $\mathrm{BD}$ based on these differences and identify neuroimaging markers that differentiated $\mathrm{BD}$ subtypes. To our knowledge, ours is the first study to identify cortical regions with significantly different $\mathrm{SA}$ values, which can be used for distinguishing $\mathrm{BD}$ subtypes. 
Table 5. Post-hoc comparison of cortical surface area between patients with BD I and BD II and HCs

\begin{tabular}{|c|c|c|c|c|}
\hline \multirow{2}{*}{ Cortical regions } & BD I vs. HC & BD II vs. HC & BD I vs. BD II & \multirow{2}{*}{ Comparison } \\
\hline & $\mathrm{p}$ & $\mathrm{p}$ & $\mathrm{p}$ & \\
\hline \multicolumn{5}{|l|}{ Left hemisphere } \\
\hline L Pars orbitalis & $5.29 \times 10^{-4}$ & $1.23 \times 10^{-9}$ & 0.063 & $\mathrm{BD}$ I \& $\mathrm{BD}$ II $<\mathrm{HC}$ \\
\hline L Long insular gyrus & $5.57 \times 10^{-5}$ & $6.86 \times 10^{-15}$ & 0.003 & BD I \& BD II $<\mathrm{HC}$ \\
\hline \multicolumn{5}{|l|}{ Right hemisphere } \\
\hline R Frontomarginal gyrus & 0.018 & $7.36 \times 10^{-6}$ & 0.141 & $\mathrm{BD}$ II $>\mathrm{HC}$ \\
\hline R Subcentral gyrus & $5.76 \times 10^{-5}$ & $1.04 \times 10^{-5}$ & 0.915 & $\mathrm{BD}$ I \& $\mathrm{BD}$ II $<\mathrm{HC}$ \\
\hline R Anterior cingulate gyrus & $4.86 \times 10^{-5}$ & $2.44 \times 10^{-8}$ & 0.387 & $\mathrm{BD}$ I \& $\mathrm{BD}$ II $<\mathrm{HC}$ \\
\hline R Posterior mid-cingulate gyrus & $2.77 \times 10^{-4}$ & $1.38 \times 10^{-6}$ & 0.541 & $\mathrm{BD}$ I \& BD II $<\mathrm{HC}$ \\
\hline R Ventral posterior cingulate gyrus & $4.22 \times 10^{-4}$ & $6.73 \times 10^{-5}$ & 0.997 & $\mathrm{BD}$ I \& BD II $>\mathrm{HC}$ \\
\hline R Cuneus & $1.43 \times 10^{-4}$ & $6.11 \times 10^{-6}$ & 0.845 & $\mathrm{BD}$ I \& $\mathrm{BD}$ II $<\mathrm{HC}$ \\
\hline R Pars opercularis & 0.005 & $3.27 \times 10^{-6}$ & 0.234 & $\mathrm{BD} \mathrm{II}<\mathrm{HC}$ \\
\hline $\mathrm{R}$ Pars triangularis & $1.71 \times 10^{-4}$ & $1.30 \times 10^{-5}$ & 0.921 & $\mathrm{BD}$ I \& $\mathrm{BD}$ II $>\mathrm{HC}$ \\
\hline R Middle frontal gyrus & $4.31 \times 10^{-4}$ & $1.01 \times 10^{-4}$ & 0.944 & $\mathrm{BD}$ I \& $\mathrm{BD}$ II $>\mathrm{HC}$ \\
\hline R Superior frontal gyrus & $3.41 \times 10^{-4}$ & $6.35 \times 10^{-5}$ & 0.970 & $\mathrm{BD}$ I \& $\mathrm{BD}$ II $<\mathrm{HC}$ \\
\hline R Long insular gyrus & 0.977 & $1.12 \times 10^{-5}$ & $6.57 \times 10^{-4}$ & $\mathrm{BD}$ II $<$ BD I \& HC \\
\hline R Superior occipital gyrus & 0.109 & $1.01 \times 10^{-5}$ & 0.037 & $\mathrm{BD}$ II $>\mathrm{HC}$ \\
\hline R Lingual gyrus & $2.80 \times 10^{-4}$ & $1.33 \times 10^{-4}$ & 0.816 & BD I \& BD II $<\mathrm{HC}$ \\
\hline R Parahippocampal gyrus & 0.006 & $3.35 \times 10^{-5}$ & 0.392 & $\mathrm{BD} \mathrm{II}>\mathrm{HC}$ \\
\hline R Precuneus & $4.58 \times 10^{-4}$ & $3.65 \times 10^{-4}$ & 0.746 & $\mathrm{BD}$ I \& BD II $<\mathrm{HC}$ \\
\hline R Straight gyrus & $2.68 \times 10^{-5}$ & $6.72 \times 10^{-9}$ & 0.352 & $\mathrm{BD}$ I \& BD II $<\mathrm{HC}$ \\
\hline R Anterior transverse temporal gyrus & $3.11 \times 10^{-4}$ & $1.93 \times 10^{-4}$ & 0.777 & $\mathrm{BD}$ I \& $\mathrm{BD}$ II $>\mathrm{HC}$ \\
\hline R Lateral superior temporal gyrus & $8.15 \times 10^{-5}$ & $3.95 \times 10^{-6}$ & 0.885 & BD I \& BD II $<\mathrm{HC}$ \\
\hline R Planum temporale & $1.08 \times 10^{-3}$ & $3.10 \times 10^{-4}$ & 0.943 & $\mathrm{BD}$ II $>\mathrm{HC}$ \\
\hline
\end{tabular}

The general linear model (GLM) was adjusted for age, sex, educational level, and intracranial volume as covariates. Regions that remained significant after Bonferroni correction are listed: 76 regions of cortex, corrected $\mathrm{p}=(0.05 / 76)=6.58 \times 10^{-4}$. BD I, bipolar disorder type I; BD II, bipolar disorder type II; HC, healthy controls; SD, standard deviation

\section{Common cortical abnormalities in $\mathrm{BD}$ patients}

Our main findings were as follows: patients with BD I and II share widespread cortical thinning in the bilateral frontal, temporal, parietal, and occipital regions; cingulate gyrus; and left insula; decreased SA in the left insula, right ACC, middle cingulate cortex, left pars orbitalis, and right superior frontal gyrus; and increased SA in the right pars triangularis, right posterior cingulate cortex, right middle frontal gyrus, and right anterior temporal gyrus compared to HCs. Our results of widespread cortical thinning are consistent with those of previous studies. ${ }^{10,11,15,40}$ Alternatively, our significant findings regarding cortical SA are contrary to those of previous studies, ${ }^{11,17-20}$ which failed to detect significant differences in SA between BD patients and HCs; however, there are a few studies that have reported results supporting our findings. ${ }^{8,13,21,22}$

The largest decrease in CT in patients with $\mathrm{BD}$ was found in the bilateral ACC, consistent with the findings of previous studies. ${ }^{10,11,15,40}$ Contrary to previous null findings, ${ }^{11,16-20}$ we found significantly decreased SA in the right ACC. Considering that the ACC is a critical region that integrates cognitive and emotional functions, shifts attention, and supports goal-directed behavior, ${ }^{16,41-44}$ we postulate that structural changes in the ACC could result in impaired cognitive control and emotion dysregulation in patients with $\mathrm{BD} .^{34,45,46}$

\section{Distinct cortical abnormalities between BD subtypes}

We found no significant differences in CT between patients with BD I and BD II, which is supported by the findings of a previous study. ${ }^{11}$ However, we found that in patients with BD I, more regions showed cortical thinning in the ventral prefrontal cortex (vPFC), including the VLPFC and OFC, which have long been studied as a critical focus in the pathophysiology of BD. ${ }^{47-50}$ While cortical thinning involved more areas in patients with $\mathrm{BD}$ I, wide areas with significant alterations in SA were observed in patients with BD II. Hibar et al. ${ }^{11}$ suggested that BD may be associated with reduced neuron numbers, but this does 
Table 6. Correlation of illness duration and HDRS score with cortical thickness in patients with BD

\begin{tabular}{|c|c|c|c|c|}
\hline \multirow[t]{2}{*}{ Cortical regions } & \multicolumn{2}{|c|}{$\begin{array}{c}\text { Illness } \\
\text { duration } \\
\text { (months) }\end{array}$} & \multicolumn{2}{|c|}{ HDRS } \\
\hline & $\mathrm{R}$ & $\mathrm{P}$ & $\mathrm{R}$ & $\mathrm{P}$ \\
\hline \multicolumn{5}{|l|}{ Left hemisphere } \\
\hline L Frontomarginal gyrus & -0.153 & 0.207 & -0.221 & 0.729 \\
\hline L Inferior occipital gyrus & -0.014 & 0.911 & -0.234 & 0.198 \\
\hline L Subcentral gyrus & 0.049 & 0.687 & -0.203 & 0.860 \\
\hline L Transverse frontopolar gyrus & -0.094 & 0.437 & -0.109 & 0.600 \\
\hline L Anterior cingulate gyrus & -0.034 & 0.782 & -0.390 & 0.138 \\
\hline L Anterior mid-cingulate gyrus & 0.008 & 0.950 & -0.294 & 0.235 \\
\hline L Dorsal posterior cingulate gyrus & s 0.020 & 0.871 & -0.362 & 0.193 \\
\hline $\begin{array}{l}\text { L Ventral posterior cingulate } \\
\text { gyrus }\end{array}$ & 0.047 & 0.702 & -0.350 & 0.115 \\
\hline L Cuneus & -0.082 & 0.498 & 0.240 & 0.502 \\
\hline L Pars opercularis & 0.045 & 0.712 & -0.181 & 0.621 \\
\hline L Pars orbitalis & -0.050 & 0.679 & -0.199 & 0.334 \\
\hline L Pars triangularis & 0.068 & 0.578 & -0.147 & 0.810 \\
\hline L Short insular gyrus & 0.096 & 0.432 & -0.235 & 0.609 \\
\hline L Middle occipital gyrus & 0.094 & 0.437 & -0.323 & 0.123 \\
\hline L Lateral occipito-temporal gyrus & 0.141 & 0.244 & -0.272 & 0.319 \\
\hline L Lingual gyrus & -0.088 & 0.469 & 0.246 & 0.445 \\
\hline L Parahippocampal gyrus & 0.021 & 0.865 & -0.298 & 0.088 \\
\hline L Angular gyrus & 0.066 & 0.588 & -0.192 & 0.711 \\
\hline L Supramarginal gyrus & 0.122 & 0.313 & -0.310 & 0.152 \\
\hline L Superior parietal lobule & 0.166 & 0.169 & -0.181 & 0.820 \\
\hline L Precentral gyrus & 0.100 & 0.411 & -0.266 & 0.135 \\
\hline L Subcallosal gyrus & -0.120 & 0.323 & -0.281 & 0.108 \\
\hline $\begin{array}{l}\text { L Anterior transverse temporal } \\
\text { gyrus }\end{array}$ & -0.090 & 0.458 & 0.268 & 0.159 \\
\hline L Planum polare & 0.058 & 0.633 & -0.252 & 0.748 \\
\hline L Middle temporal gyrus & 0.061 & 0.617 & -0.277 & 0.413 \\
\hline \multicolumn{5}{|l|}{ Right hemisphere } \\
\hline R Frontomarginal gyrus & 0.144 & 0.234 & 0.107 & 0.378 \\
\hline R Inferior occipital gyrus & 0.034 & 0.778 & -0.077 & 0.527 \\
\hline R Subcentral gyrus & 0.036 & 0.769 & -0.161 & 0.184 \\
\hline $\mathrm{R}$ Transverse frontopolar gyrus & 0.001 & 0.996 & 0.161 & 0.183 \\
\hline R Anterior cingulate gyrus & -0.027 & 0.825 & -0.010 & 0.937 \\
\hline R Anterior mid-cingulate gyrus & 0.101 & 0.404 & -0.228 & 0.058 \\
\hline $\begin{array}{l}\text { R Dorsal posterior cingulate } \\
\text { gyrus }\end{array}$ & 0.258 & 0.031 & -0.030 & 0.804 \\
\hline $\begin{array}{l}\text { R Ventral posterior cingulate } \\
\text { gyrus }\end{array}$ & 0.022 & 0.853 & -0.089 & 0.464 \\
\hline R Cuneus & -0.064 & 0.599 & 0.106 & 0.382 \\
\hline R Pars opercularis & -0.084 & 0.491 & -0.032 & 0.790 \\
\hline
\end{tabular}

Table 6. Correlation of illness duration and HDRS score with cortical thickness in patients with BD (continued)

\begin{tabular}{|c|c|c|c|c|}
\hline \multirow[t]{2}{*}{ Cortical regions } & \multicolumn{2}{|c|}{$\begin{array}{c}\text { Illness } \\
\text { duration } \\
\text { (months) }\end{array}$} & \multicolumn{2}{|c|}{ HDRS } \\
\hline & $\mathrm{R}$ & $\mathrm{P}$ & $\mathrm{R}$ & $\mathrm{P}$ \\
\hline R Pars orbitalis & -0.006 & 0.959 & -0.063 & 0.603 \\
\hline $\mathrm{R}$ Pars triangularis & -0.115 & 0.344 & 0.108 & 0.373 \\
\hline R Middle frontal gyrus & 0.007 & 0.957 & 0.027 & 0.827 \\
\hline R Middle occipital gyrus & 0.109 & 0.367 & -0.121 & 0.320 \\
\hline R Lateral occipito-temporal gyrus & 0.040 & 0.740 & -0.138 & 0.255 \\
\hline R Lingual gyrus & -0.070 & 0.563 & 0.136 & 0.263 \\
\hline R Parahippocampal gyrus & 0.132 & 0.277 & -0.176 & 0.144 \\
\hline R Orbital gyrus & 0.016 & 0.895 & 0.002 & 0.985 \\
\hline R Angular gyrus & -0.055 & 0.650 & -0.075 & 0.540 \\
\hline R Supramarginal gyrus & 0.028 & 0.815 & -0.171 & 0.157 \\
\hline R Superior parietal lobule & 0.154 & 0.202 & -0.110 & 0.366 \\
\hline R Precentral gyrus & 0.031 & 0.801 & -0.075 & 0.539 \\
\hline R Planum polare & 0.058 & 0.631 & -0.171 & 0.156 \\
\hline R Middle temporal gyrus & 0.071 & 0.557 & -0.055 & 0.652 \\
\hline
\end{tabular}

A two-tailed Pearson's partial correlation analysis was performed that controlled for age, sex, educational level, and intracranial volume. The 49 cortical regions that remained significant after Bonferroni correction are listed (corrected $\mathrm{p}=[0.05 / 49]=1.02 \times 10^{-3}$ ). BD, bipolar disorder; HDRS, Hamilton Depression Rating Scale

not lead to changes in overall cortical size from the result that BD showed significant changes only in CT, not in SA, in their study. However, our study showed that investigating both CT and SA enhanced the sensitivity for distinguishing BD subtypes. As previous research ${ }^{11}$ focused on the comparison of cortical abnormalities between patients with $\mathrm{BD}$ and HCs, comparisons between patients with $\mathrm{BD} I$ and $\mathrm{HCs}$ and between patients with BD II and HCs were not performed, although post-hoc analyses were performed for differences according to subtype. ${ }^{11}$ Therefore, we speculate that the difference of our study may be a result of considering $\mathrm{BD}$ subtypes as different disease entities and performing three-group comparisons, which helped to elucidate subtype-specific morphological parameters.

Interestingly, the right long insula was found to be a significant region for differentiating BD subtypes even after Bonferroni correction. Patients with BD II demonstrated a smaller SA than patients with $\mathrm{BD}$ I in the right long insula, which is posterior part of the insula. Similarly, a smaller SA in the left posterior insula was detected in patients with BD II compared to that in patients with BD I, but this did not remain significant after correction for multiple comparisons. Although the anterior insula has been suggested as a critical multimodal hub within the cingulo-opercular system (i.e., the salience network) involved in detecting emotional salience and integrating sensorimotor, 
Table 7. Correlation of illness duration and HDRS score with cortical surface areas in patients with BD

\begin{tabular}{|c|c|c|c|c|}
\hline \multirow[t]{2}{*}{ Cortical regions } & \multicolumn{2}{|c|}{$\begin{array}{c}\text { Illness } \\
\text { duration } \\
\text { (months) }\end{array}$} & \multicolumn{2}{|c|}{ HDRS } \\
\hline & $\mathrm{R}$ & $\mathrm{P}$ & $\mathrm{R}$ & $\mathrm{P}$ \\
\hline \multicolumn{5}{|l|}{ Left hemisphere } \\
\hline L Pars orbitalis & -0.002 & 0.990 & -0.111 & 0.359 \\
\hline L Long insular gyrus & -0.076 & 0.531 & -0.250 & 0.037 \\
\hline \multicolumn{5}{|l|}{ Right hemisphere } \\
\hline R Frontomarginal gyrus & -0.174 & 0.149 & -0.007 & 0.956 \\
\hline R Subcentral gyrus & 0.098 & 0.419 & 0.085 & 0.483 \\
\hline R Transverse frontopolar gyrus & -0.065 & 0.593 & -0.008 & 0.945 \\
\hline R Anterior cingulate gyrus & -0.009 & 0.938 & -0.164 & 0.174 \\
\hline R Posterior mid-cingulate gyrus & 0.035 & 0.774 & -0.195 & 0.106 \\
\hline $\begin{array}{l}\text { R Ventral posterior cingulate } \\
\text { gyrus }\end{array}$ & -0.071 & 0.561 & 0.043 & 0.725 \\
\hline R Cuneus & 0.066 & 0.586 & -0.020 & 0.868 \\
\hline R Pars opercularis & 0.204 & 0.091 & -0.162 & 0.181 \\
\hline R Pars orbitalis & -0.152 & 0.210 & 0.098 & 0.420 \\
\hline $\mathrm{R}$ Pars triangularis & -0.080 & 0.510 & 0.096 & 0.430 \\
\hline R Middle frontal gyrus & -0.118 & 0.329 & 0.161 & 0.184 \\
\hline R Superior frontal gyrus & 0.079 & 0.517 & -0.071 & 0.559 \\
\hline $\mathrm{R}$ Long insular gyrus & -0.064 & 0.596 & -0.292 & 0.014 \\
\hline R Short insular gyrus & -0.123 & 0.310 & 0.044 & 0.718 \\
\hline R Superior occipital gyrus & -0.001 & 0.991 & 0.104 & 0.393 \\
\hline R Lingual gyrus & 0.102 & 0.399 & -0.006 & 0.959 \\
\hline R Parahippocampal gyrus & -0.114 & 0.349 & 0.115 & 0.344 \\
\hline R Precuneus & 0.050 & 0.678 & 0.026 & 0.829 \\
\hline R Straight gyrus & 0.037 & 0.758 & -0.145 & 0.232 \\
\hline $\begin{array}{l}\text { R Anterior transverse temporal } \\
\text { gyrus }\end{array}$ & -0.084 & 0.489 & 0.084 & 0.490 \\
\hline R Lateral superior temporal gyrus & 0.108 & 0.373 & -0.116 & 0.337 \\
\hline R Planum temporale & -0.108 & 0.375 & 0.071 & 0.557 \\
\hline
\end{tabular}

A two-tailed Pearson's partial correlation analysis was performed that controlled for age, sex, educational level, and intracranial volume. The 24 cortical regions that remained significant after Bonferroni correction are listed (corrected $\mathrm{p}=[0.05 / 24]=2.08 \times 10^{-3}$ ). $\mathrm{BD}$, bipolar disorder; HDRS, Hamilton Depression Rating Scale

emotional, and cognitive information, ${ }^{51,52}$ relatively little research has been conducted on the posterior insula. Three insular subregions, the posterior, ventral anterior, and dorsal anterior regions, ${ }^{53}$ showed different connectivity patterns in functional MRI studies. ${ }^{52,54}$ Deen et al..$^{54}$ suggested the possibility that the dorsal anterior insula/dorsal ACC network creates a pathway through which inputs, such as somatosensory and interoceptive information from the posterior insula or affective information from the ventral anterior insula, can affect decision-making and be- havior. Therefore, we speculate that prominent deformities in the posterior insula contribute to dysfunction in the integration of emotional and cognitive signals through impaired somatosensory and interoceptive input in patients with BD II.

To our knowledge, no previous studies have suggested that the right posterior insula can be used to differentiate between BD subtypes; however, several functional MRI studies ${ }^{52,55}$ have focused on the insular region to distinguish bipolar depression from unipolar depression. Ambrosi et al..$^{52}$ found that functional connectivity between the left posterior insula and right frontopolar prefrontal cortex could be a biomarker distinguishing between bipolar and unipolar depression, as functional connectivity was found to be lower in bipolar depression than in unipolar depression. Although such previous studies have been conducted on patients with BD I or BD II indiscriminately, we suggested that connectivity with the posterior insula could be more prominent in those with BD II; however, further investigations are needed to elucidate its role in the pathophysiology of $\mathrm{BD}$.

Additionally, we explored the correlation of clinical states and illness duration with CT and SA but did not find any significant findings after Bonferroni correction. A previous study reported findings consistent with our results, showing no association between a euthymic or depressive state and cortical abnormalities. ${ }^{11}$ Although there was no association between illness duration and cortical abnormalities in our study, a previous study reported that a longer illness duration in $\mathrm{BD}$ patients was positively associated with CT in the occipital, parietal, and right frontal cortices, but not with SA. ${ }^{11}$ Therefore, further studies are required to address this issue.

There are several limitations to our study. First, due to the cross-sectional design, we could not prove causality between cortical abnormalities and BD subtypes. Second, the BD groups were heterogeneous in terms of medications used, including lithium, antiepileptic drugs (AEDs), atypical antipsychotics (AAs), and antidepressants (ADs). To overcome this limitation, we analyzed the effect of lithium, AEDs, and AAs on cortical abnormalities in our additional analyses (Supplementary Tables 7-12 in the online-only Data Supplement). We found that the use of AAs was significantly associated with cortical thinning in the right pars triangularis (AA users: $2.57 \pm 0.17$; AA nonusers: $\left.2.80 \pm 0.14 ; \mathrm{F}=15.858, \mathrm{p}=1.69 \times 10^{-4}\right)$, but the other results were insignificant (lithium or AED use with respect to CT or SA; AA use with respect to SA). Contrarily, a meta-analysis found evidence of increased CT or SA with the use of lithium, cortical thinning with the use of AEDs, and reduced SA with the use of AAs in several regions. ${ }^{11,15}$ This suggests that the effect of medication on cortical abnormalities may vary and is dependent on sample size. As we did not control for the dosage and combination effects of various medications, we could 
not overlook the possible confounding effects of medications. Third, our sample was heterogeneous with regard to mood state as more patients with $\mathrm{BD}$ II were in a depressive state than those with BD I. This could lead to the misconception that our differences in SA in the right long insula may be due to the depressive state. However, large-scale studies, such as the ENIGMA consortium, have investigated cortical SA in adult patients with major depressive disorder but showed no SA abnormalities, including in the insular gyrus, when compared with controls. ${ }^{56}$ Fourth, although we suggested that a decreased SA in the right long insula is crucial for differentiating between the $\mathrm{BD}$ subtypes, we failed to prove the correlation of SA in the right long insula with clinical states and illness duration after Bonferroni correction. However, our result was consistent with that reported by a previous study that found no significant correlation between clinical state and illness duration with SA. CT and SA are genetically independent. Unlike cortical thinning, which begins at 2-4 years and gradually progresses throughout lifetime, cortical SA expands until 12 years and remains relatively stable..$^{56-60}$ Therefore, we hypothesized that SA is less affected by environmental factors, such as disease state and duration, because it might be determined earlier in the neurodevelopmental period. ${ }^{12,14}$

Despite several limitations, our study is one of the few that explored common and unique cortical abnormalities in patients with $\mathrm{BD}$ I and $\mathrm{BD}$ II. Our finding of cortical thinning in patients with $\mathrm{BD}$ is consistent with previous findings involving the bilateral frontal, temporal, and occipital regions; cingulate gyrus; and insula. We found alterations in SA in BD patients, including increase SA in the pars triangularis and decreased SA in the insula. Our study confirms previous results and provides novel findings for comparing $\mathrm{BD}$ subtypes. Cortical thinning involved more areas, including the $\mathrm{PFC}$, in patients with $\mathrm{BD}$ I, while changes in SA were more extensive in patients with BD II.

In conclusion, our finding of decreased SA in the right long insula could be crucial for differentiating between BD subtypes. Further large-scale and longitudinal investigations regarding the association between BD subtypes and cortical abnormalities are necessary for confirmation.

\section{Supplementary Materials}

The online-only Data Supplement is available with this article at https://doi.org/10.30773/pi.2021.0074.

\section{Availability of Data and Material}

All data generated or analyzed during the study are included in this published article (and its supplementary information files).

\section{Conflicts of Interest}

The authors have no potential conflicts of interest to disclose.

\section{Author Contributions}

Conceptualization: Byung-Joo Ham. Data curation: Wooyoung Kang, Youbin Kang, Aram Kim. Formal analysis: Kyu-Man Han, Woo-Suk Tae. Funding acquisition: Byung-Joo Ham. Investigation: Byung-Joo Ham. Methodology: Kyu-Man Han, Woo-Suk Tae. Project administration: Byung-Joo Ham. Resources: Byung-Joo Ham. Software: Woo-Suk Tae. Supervision: Byung-Joo Ham. Validation: Kyu-Man Han. Visualization: Kyu-Man Han. Writing_original draft: Yoonmi Woo. Writing_review \& editing: ByungJoo Ham, Kyu-Man Han.

\section{ORCID iDs}

Yoonmi Woo Wooyoung Kang

Youbin Kang

Aram Kim

Kyu-Man Han

Woo-Suk Tae

Byung-Joo Ham

https://orcid.org/0000-0001-7431-000X

https://orcid.org/0000-0003-4733-027X https://orcid.org/0000-0002-6173-9920

https://orcid.org/0000-0002-3941-218X https://orcid.org/0000-0002-1982-4216 https://orcid.org/0000-0003-0451-0713 https://orcid.org/0000-0002-0108-2058

\section{Funding Statement}

This work was supported by the National Research Foundation of Korea (NRF) funded by the Ministry of Education, Science and Technology (NRF2020M3E5D9080792).

\section{REFERENCES}

1. American Psychiatric Association. Diagnostic and Statistical Manual of Mental Disorders (DSM- $5^{\circledR}$ ). Washington DC: American Psychiatric Pub; 2013.

2. Grande I, Berk M, Birmaher B, Vieta E. Bipolar disorder. Lancet 2016; 387:1561-1572.

3. Ferrari AJ, Stockings E, Khoo JP, Erskine HE, Degenhardt L, Vos T, et al. The prevalence and burden of bipolar disorder: findings from the Global Burden of Disease Study 2013. Bipolar Disord 2016;18:440-450.

4. Sadock BJ, Sadock VA. Kaplan and Sadock's Synopsis of Psychiatry: Behavioral Sciences/Clinical Psychiatry. Philadephila: Lippincott Williams \& Wilkins; 2011.

5. Rihmer Z, Pestality P. Bipolar II disorder and suicidal behavior. Psychiatr Clin North Am 1999;22:667-673, ix-x.

6. Maina G, Albert U, Bellodi L, Colombo C, Faravelli C, Monteleone P, et al. Health-related quality of life in euthymic bipolar disorder patients: differences between bipolar I and II subtypes. J Clin Psychiatry 2007; 68:207-212.

7. Ha TH, Ha K, Kim JH, Choi JE. Regional brain gray matter abnormalities in patients with bipolar II disorder: a comparison study with bipolar I patients and healthy controls. Neurosci Lett 2009;456:44-48.

8. Abe C, Ekman CJ, Sellgren C, Petrovic P, Ingvar M, Landen M. Cortical thickness, volume and surface area in patients with bipolar disorder types I and II. J Psychiatry Neurosci 2016;41:240-250.

9. Winkler AM, Kochunov P, Blangero J, Almasy L, Zilles K, Fox PT, et al. Cortical thickness or grey matter volume? The importance of selecting the phenotype for imaging genetics studies. Neuroimage 2010;53:11351146.

10. Hanford LC, Nazarov A, Hall GB, Sassi RB. Cortical thickness in bipolar disorder: a systematic review. Bipolar Disord 2016;18:4-18.

11. Hibar DP, Westlye LT, Doan NT, Jahanshad N, Cheung JW, Ching CRK, et al. Cortical abnormalities in bipolar disorder: an MRI analysis of 6503 individuals from the ENIGMA Bipolar Disorder Working Group. Mol Psychiatry 2018;23:932-942.

12. Rakic P. Specification of cerebral cortical areas. Science 1988;241:170176.

13. Hartberg CB, Sundet K, Rimol LM, Haukvik UK, Lange EH, Nesvag R, et al. Brain cortical thickness and surface area correlates of neurocognitive performance in patients with schizophrenia, bipolar disorder, 
and healthy adults. J Int Neuropsychol Soc 2011;17:1080-1093.

14. Habets P, Marcelis M, Gronenschild E, Drukker M, van Os J. Reduced cortical thickness as an outcome of differential sensitivity to environmental risks in schizophrenia. Biol Psychiatry 2011;69:487-494.

15. Ching CRK, Hibar DP, Gurholt TP, Nunes A, Thomopoulos SI, Abe C, et al. What we learn about bipolar disorder from large-scale neuroimaging: Findings and future directions from the ENIGMA Bipolar Disorder Working Group. Hum Brain Mapp 2020 [Online ahead of print].

16. Fornito A, Malhi GS, Lagopoulos J, Ivanovski B, Wood SJ, Saling MM, et al. Anatomical abnormalities of the anterior cingulate and paracingulate cortex in patients with bipolar I disorder. Psychiatry Res 2008; 162:123-132.

17. Rimol LM, Nesvag R, Hagler DJ Jr, Bergmann O, Fennema-Notestine C, Hartberg CB, et al. Cortical volume, surface area, and thickness in schizophrenia and bipolar disorder. Biol Psychiatry 2012;71:552-560.

18. Elvsåshagen T, Westlye LT, Bøen E, Hol PK, Andreassen OA, Boye B, et al. Bipolar II disorder is associated with thinning of prefrontal and temporal cortices involved in affect regulation. Bipolar Disord 2013;15: 855-864.

19. Janssen J, Aleman-Gomez Y, Schnack H, Balaban E, Pina-Camacho L, Alfaro-Almagro F, et al. Cortical morphology of adolescents with bipolar disorder and with schizophrenia. Schizophr Res 2014;158:91-99.

20. Roberts G, Lenroot R, Frankland A, Yeung PK, Gale N, Wright A, et al. Abnormalities in left inferior frontal gyral thickness and parahippocampal gyral volume in young people at high genetic risk for bipolar disorder. Psychol Med 2016;46:2083-2096.

21. Fung G, Deng Y, Zhao Q, Li Z, Qu M, Li K, et al. Distinguishing bipolar and major depressive disorders by brain structural morphometry: a pilot study. BMC Psychiatry 2015;15:298.

22. Yalin N, Saricicek A, Hidiroglu C, Zugman A, Direk N, Ada E, et al. Cortical thickness and surface area as an endophenotype in bipolar disorder type I patients and their first-degree relatives. Neuroimage Clin 2019;22:101695.

23. Lyoo IK, Sung YH, Dager SR, Friedman SD, Lee JY, Kim SJ, et al. Regional cerebral cortical thinning in bipolar disorder. Bipolar Disord 2006;8:65-74.

24. Rimol LM, Hartberg CB, Nesvag R, Fennema-Notestine C, Hagler DJ Jr, Pung CJ, et al. Cortical thickness and subcortical volumes in schizophrenia and bipolar disorder. Biol Psychiatry 2010;68:41-50.

25. Maller JJ, Thaveenthiran P, Thomson RH, McQueen S, Fitzgerald PB. Volumetric, cortical thickness and white matter integrity alterations in bipolar disorder type I and II. J Affect Disord 2014;169:118-127.

26. Emrich HM. [Cognition and feelings--on the neurobiology of the perception-emotion connection]. Geburtshilfe Frauenheilkd 1996;56:M6M8.

27. Hamilton M. A rating scale for depression. J Neurol Neurosurg Psychiatry 1960;23:56-62.

28. Young RC, Biggs JT, Ziegler VE, Meyer DA. A rating scale for mania: reliability, validity and sensitivity. Br J Psychiatry 1978;133:429-435.

29. Dale AM, Fischl B, Sereno MI. Cortical surface-based analysis. I. Segmentation and surface reconstruction. Neuroimage 1999;9:179-194.

30. Fischl B, Liu A, Dale AM. Automated manifold surgery: constructing geometrically accurate and topologically correct models of the human cerebral cortex. IEEE Trans Med Imaging 2001;20:70-80.

31. Fischl B, Salat DH, Busa E, Albert M, Dieterich M, Haselgrove C, et al. Whole brain segmentation: automated labeling of neuroanatomical structures in the human brain. Neuron 2002;33:341-355.

32. Fischl B, van der Kouwe A, Destrieux C, Halgren E, Segonne F, Salat $\mathrm{DH}$, et al. Automatically parcellating the human cerebral cortex. Cereb Cortex 2004;14:11-22.

33. Segonne F, Pacheco J, Fischl B. Geometrically accurate topology-correction of cortical surfaces using nonseparating loops. IEEE Trans Med Imaging 2007;26:518-529.

34. Han KM, Choi S, Jung J, Na KS, Yoon HK, Lee MS, et al. Cortical thickness, cortical and subcortical volume, and white matter integrity in patients with their first episode of major depression. J Affect Disord 2014;155:42-48

35. Han KM, Won E, Sim Y, Kang J, Han C, Kim YK, et al. Influence of FKBP5 polymorphism and DNA methylation on structural changes of the brain in major depressive disorder. Sci Rep 2017;7:42621.

36. Han MR, Han KM, Kim A, Kang W, Kang Y, Kang J, et al. Wholeexome sequencing identifies variants associated with structural MRI markers in patients with bipolar disorders. J Affect Disord 2019;249: 159-168.

37. Destrieux C, Fischl B, Dale A, Halgren E. Automatic parcellation of human cortical gyri and sulci using standard anatomical nomenclature. Neuroimage 2010;53:1-15.

38. Shinkov AD, Borisova AM, Kovacheva RD, Vlahov YD, Dakovska LN, Atanassova ID, et al. Influence of serum levels of thyroid-stimulating hormone and anti-thyroid peroxidase antibodies, age and gender on depression as measured by the Zung Self-Rating Depression Scale. Folia Med (Plovdiv) 2014;56:24-31.

39. Turkoz I, Fu DJ, Bossie CA, Sheehan JJ, Alphs L. Relationship between the clinical global impression of severity for schizoaffective disorder scale and established mood scales for mania and depression. J Affect Disord 2013;150:17-22.

40. Phillips ML, Swartz HA. A critical appraisal of neuroimaging studies of bipolar disorder: toward a new conceptualization of underlying neural circuitry and a road map for future research. Am J Psychiatry 2014;171: 829-843.

41. Cohen JD, Botvinick M, Carter CS. Anterior cingulate and prefrontal cortex: who's in control? Nat Neurosci 2000;3:421-423.

42. Phillips ML, Drevets WC, Rauch SL, Lane R. Neurobiology of emotion perception I: the neural basis of normal emotion perception. Biol Psychiatry 2003;54:504-514.

43. Ridderinkhof KR, Ullsperger M, Crone EA, Nieuwenhuis S. The role of the medial frontal cortex in cognitive control. Science 2004;306:443447.

44. Clark DL, Boutros NN, Mendez MF. The Brain and Behavior: An Introduction to Behavioral Neuroanatomy. Cambridge: Cambridge University Press; 2010.

45. Gruber SA, Dahlgren MK, Sagar KA, Gonenc A, Norris L, Cohen BM, et al. Decreased Cingulate Cortex activation during cognitive control processing in bipolar disorder. J Affect Disord 2017;213:86-95.

46. Gruber SA, Rogowska J, Yurgelun-Todd DA. Decreased activation of the anterior cingulate in bipolar patients: an fMRI study. J Affect Disord 2004;82:191-201.

47. Lawrence NS, Williams AM, Surguladze S, Giampietro V, Brammer MJ, Andrew C, et al. Subcortical and ventral prefrontal cortical neural responses to facial expressions distinguish patients with bipolar disorder and major depression. Biol Psychiatry 2004;55:578-587.

48. Malhi GS, Lagopoulos J, Sachdev PS, Ivanovski B, Shnier R, Ketter T. Is a lack of disgust something to fear? A functional magnetic resonance imaging facial emotion recognition study in euthymic bipolar disorder patients. Bipolar Disord 2007;9:345-357.

49. Kronhaus DM, Lawrence NS, Williams AM, Frangou S, Brammer MJ, Williams SC, et al. Stroop performance in bipolar disorder: further evidence for abnormalities in the ventral prefrontal cortex. Bipolar Disord 2006;8:28-39.

50. Stanfield AC, Moorhead TW, Job DE, McKirdy J, Sussmann JE, Hall J, et al. Structural abnormalities of ventrolateral and orbitofrontal cortex in patients with familial bipolar disorder. Bipolar Disord 2009;11:135144.

51. Menon V. Large-scale brain networks and psychopathology: a unifying triple network model. Trends Cogn Sci 2011;15:483-506.

52. Ambrosi E, Arciniegas DB, Madan A, Curtis KN, Patriquin MA, Jorge $\mathrm{RE}$, et al. Insula and amygdala resting-state functional connectivity differentiate bipolar from unipolar depression. Acta Psychiatr Scand 2017;136:129-139.

53. Kulkarni J, Gavrilidis E, Hudaib AR, Bleeker C, Worsley R, Gurvich C. 
Development and validation of a new rating scale for perimenopausal depression-the Meno-D. Transl Psychiatry 2018;8:123.

54. Deen B, Pitskel NB, Pelphrey KA. Three systems of insular functional connectivity identified with cluster analysis. Cereb Cortex 2011;21: 1498-1506.

55. Ellard KK, Zimmerman JP, Kaur N, Van Dijk KRA, Roffman JL, Nierenberg AA, et al. Functional connectivity between anterior insula and key nodes of frontoparietal executive control and salience networks distinguish bipolar depression from unipolar depression and healthy control subjects. Biol Psychiatry Cogn Neurosci Neuroimaging 2018;3:473484 .

56. Schmaal L, Hibar DP, Sämann PG, Hall GB, Baune BT, Jahanshad N, et al. Cortical abnormalities in adults and adolescents with major depression based on brain scans from 20 cohorts worldwide in the ENIGMA Major Depressive Disorder Working Group. Mol Psychiatry 2017;22:
900-909.

57. Storsve AB, Fjell AM, Tamnes CK, Westlye LT, Overbye K, Aasland HW, et al. Differential longitudinal changes in cortical thickness, surface area and volume across the adult life span: regions of accelerating and decelerating change. J Neurosci 2014;34:8488-8498.

58. Wierenga LM, Langen M, Oranje B, Durston S. Unique developmental trajectories of cortical thickness and surface area. Neuroimage 2014; 87:120-126.

59. Amlien IK, Fjell AM, Tamnes CK, Grydeland H, Krogsrud SK, Chaplin TA, et al. Organizing principles of human cortical development-thickness and area from 4 to 30 years: insights from comparative primate neuroanatomy. Cereb Cortex 2016;26:257-267.

60. Jernigan TL, Brown TT, Bartsch H, Dale AM. Toward an integrative science of the developing human mind and brain: focus on the developing cortex. Dev Cogn Neurosci 2016;18:2-11. 
Supplementary Table 1. Correlation of illness duration and HDRS score with cortical thickness in patients with BD I

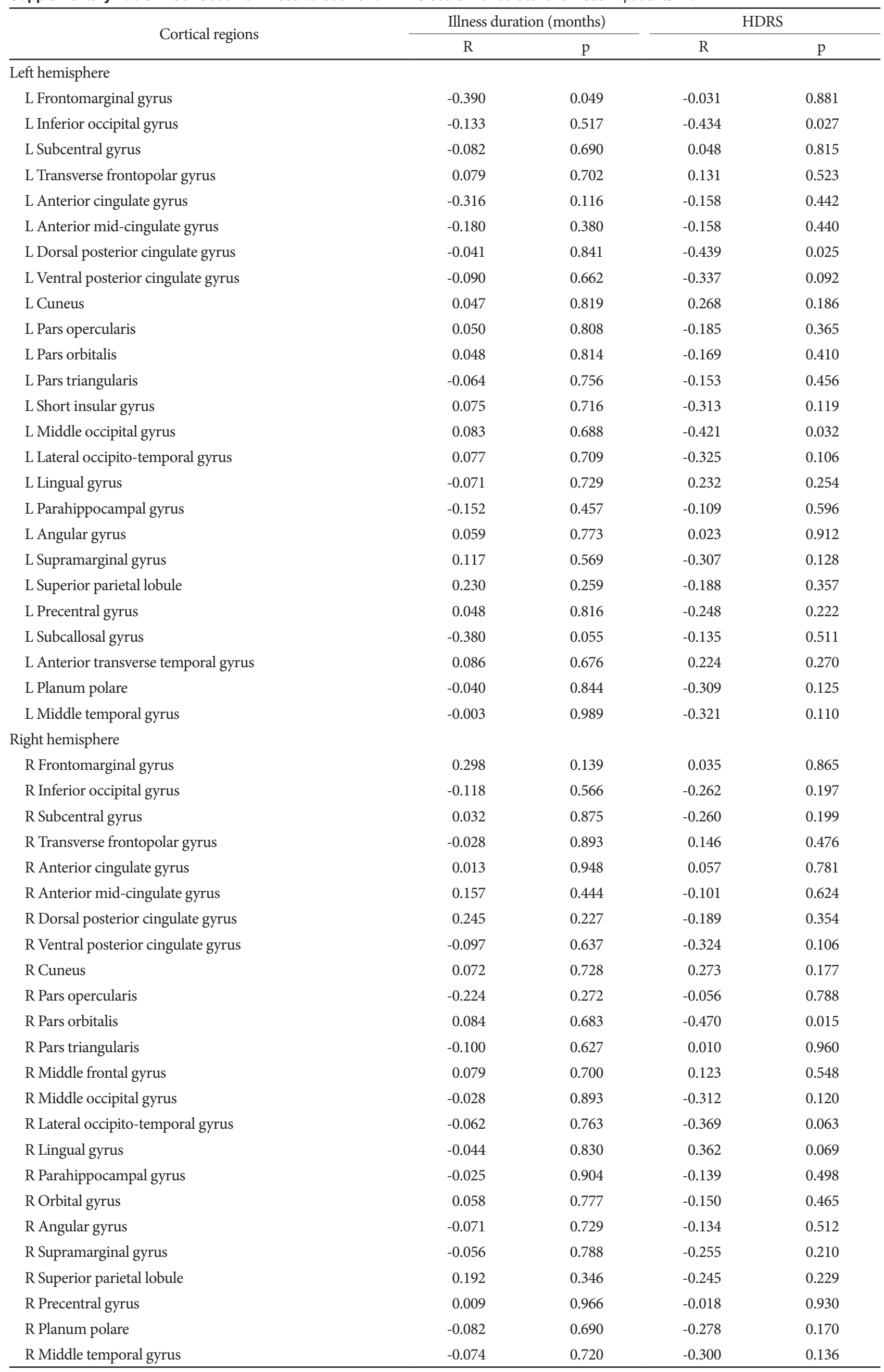

A two-tailed Pearson's partial correlation analysis was performed that controlled for age, sex, educational level, and intracranial volume. The 49 cortical regions that remained significant after Bonferroni correction are listed (corrected $\mathrm{p}=[0.05 / 49]=1.02 \times 10^{-3}$ ). $\mathrm{BD}$, bipolar disorder; HDRS, Hamilton Depression Rating Scale 
Supplementary Table 2. Correlation of illness duration and HDRS score with cortical thickness in patients with BD II

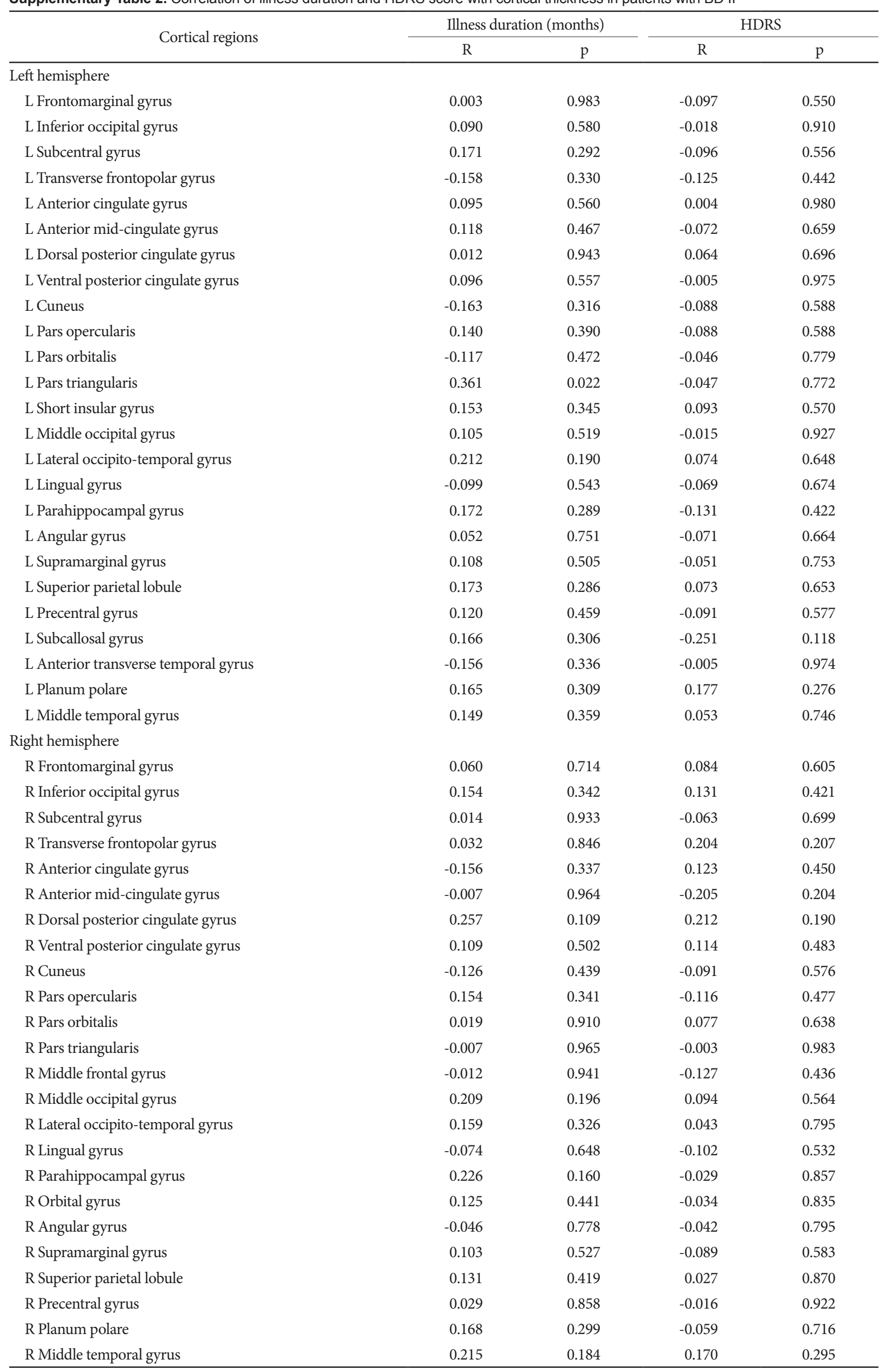

A two-tailed Pearson's partial correlation analysis was performed that controlled for age, sex, educational level, and intracranial volume. The 49 cortical regions that remained significant after Bonferroni correction are listed (corrected $\mathrm{p}=[0.05 / 49]=1.02 \times 10^{-3}$ ). $\mathrm{BD}$, bipolar disorder; HDRS, Hamilton Depression Rating Scale 
Supplementary Table 3. Correlation of illness duration and HDRS score with cortical surface area in patients with BD I

\begin{tabular}{|c|c|c|c|c|}
\hline \multirow{2}{*}{ Cortical regions } & \multicolumn{2}{|c|}{ Illness duration (months) } & \multicolumn{2}{|c|}{ HDRS } \\
\hline & $\mathrm{R}$ & $\mathrm{p}$ & $\mathrm{R}$ & $\mathrm{p}$ \\
\hline \multicolumn{5}{|l|}{ Left hemisphere } \\
\hline L Pars orbitalis & -0.298 & 0.140 & -0.271 & 0.180 \\
\hline L Long insular gyrus & -0.425 & 0.030 & -0.252 & 0.214 \\
\hline \multicolumn{5}{|l|}{ Right hemisphere } \\
\hline R Frontomarginal gyrus & -0.072 & 0.728 & 0.258 & 0.203 \\
\hline R Subcentral gyrus & -0.069 & 0.739 & -0.163 & 0.427 \\
\hline $\mathrm{R}$ Transverse frontopolar gyrus & 0.022 & 0.916 & 0.270 & 0.182 \\
\hline R Anterior cingulate gyrus & -0.093 & 0.651 & -0.414 & 0.036 \\
\hline R Posterior mid-cingulate gyrus & -0.022 & 0.914 & -0.331 & 0.099 \\
\hline $\mathrm{R}$ Ventral posterior cingulate gyrus & 0.048 & 0.817 & 0.208 & 0.307 \\
\hline R Cuneus & 0.017 & 0.934 & -0.257 & 0.205 \\
\hline R Pars opercularis & 0.066 & 0.749 & -0.302 & 0.134 \\
\hline $\mathrm{R}$ Pars orbitalis & -0.142 & 0.488 & 0.351 & 0.079 \\
\hline $\mathrm{R}$ Pars triangularis & 0.018 & 0.929 & 0.378 & 0.057 \\
\hline R Middle frontal gyrus & -0.018 & 0.931 & 0.343 & 0.086 \\
\hline R Superior frontal gyrus & -0.039 & 0.851 & -0.331 & 0.099 \\
\hline R Long insular gyrus & -0.339 & 0.090 & -0.188 & 0.358 \\
\hline R Short insular gyrus & -0.043 & 0.833 & 0.236 & 0.245 \\
\hline R Superior occipital gyrus & 0.178 & 0.385 & 0.135 & 0.512 \\
\hline R Lingual gyrus & -0.012 & 0.954 & -0.254 & 0.210 \\
\hline R Parahippocampal gyrus & 0.020 & 0.924 & 0.361 & 0.070 \\
\hline R Precuneus & -0.030 & 0.883 & -0.209 & 0.304 \\
\hline R Straight gyrus & -0.107 & 0.602 & -0.415 & 0.035 \\
\hline R Anterior transverse temporal gyrus & -0.005 & 0.981 & 0.292 & 0.148 \\
\hline R Lateral superior temporal gyrus & 0.090 & 0.662 & -0.361 & 0.070 \\
\hline R Planum temporale & -0.029 & 0.888 & 0.219 & 0.282 \\
\hline
\end{tabular}

A two-tailed Pearson's partial correlation analysis was performed that controlled for age, sex, educational level, and intracranial volume. The 24 cortical regions that remained significant after Bonferroni correction are listed (corrected $\mathrm{p}=[0.05 / 24]=2.08 \times 10^{-3}$ ). $\mathrm{BD}$, bipolar disorder; HDRS, Hamilton Depression Rating Scale 
Supplementary Table 4. Correlation of illness duration and HDRS score with cortical surface area in patients with BD II

\begin{tabular}{|c|c|c|c|c|}
\hline \multirow{2}{*}{ Cortical regions } & \multicolumn{2}{|c|}{ Illness duration (months) } & \multicolumn{2}{|c|}{ HDRS } \\
\hline & $\mathrm{R}$ & $\mathrm{p}$ & $\mathrm{R}$ & $\mathrm{p}$ \\
\hline \multicolumn{5}{|l|}{ Left hemisphere } \\
\hline L Pars orbitalis & 0.143 & 0.379 & 0.135 & 0.405 \\
\hline L Long insular gyrus & -0.035 & 0.830 & 0.013 & 0.937 \\
\hline \multicolumn{5}{|l|}{ Right hemisphere } \\
\hline R Frontomarginal gyrus & -0.168 & 0.299 & -0.202 & 0.211 \\
\hline R Subcentral gyrus & 0.249 & 0.121 & 0.264 & 0.100 \\
\hline $\mathrm{R}$ Transverse frontopolar gyrus & -0.142 & 0.381 & -0.208 & 0.198 \\
\hline R Anterior cingulate gyrus & 0.021 & 0.899 & 0.081 & 0.620 \\
\hline R Posterior mid-cingulate gyrus & 0.061 & 0.708 & -0.030 & 0.856 \\
\hline $\mathrm{R}$ Ventral posterior cingulate gyrus & -0.167 & 0.302 & -0.126 & 0.437 \\
\hline R Cuneus & 0.111 & 0.496 & 0.168 & 0.301 \\
\hline R Pars opercularis & 0.280 & 0.080 & 0.031 & 0.848 \\
\hline $\mathrm{R}$ Pars orbitalis & -0.180 & 0.268 & -0.083 & 0.611 \\
\hline $\mathrm{R}$ Pars triangularis & -0.149 & 0.358 & -0.110 & 0.500 \\
\hline R Middle frontal gyrus & -0.209 & 0.196 & -0.038 & 0.817 \\
\hline R Superior frontal gyrus & 0.165 & 0.310 & 0.161 & 0.320 \\
\hline R Long insular gyrus & -0.174 & 0.283 & -0.013 & 0.935 \\
\hline R Short insular gyrus & -0.195 & 0.227 & -0.121 & 0.456 \\
\hline R Superior occipital gyrus & -0.049 & 0.765 & 0.000 & 1.000 \\
\hline R Lingual gyrus & 0.215 & 0.183 & 0.205 & 0.205 \\
\hline R Parahippocampal gyrus & -0.193 & 0.232 & -0.133 & 0.412 \\
\hline R Precuneus & 0.140 & 0.389 & 0.212 & 0.190 \\
\hline R Straight gyrus & 0.096 & 0.557 & 0.139 & 0.391 \\
\hline R Anterior transverse temporal gyrus & -0.170 & 0.294 & -0.091 & 0.578 \\
\hline R Lateral superior temporal gyrus & 0.108 & 0.506 & 0.083 & 0.610 \\
\hline R Planum temporale & -0.168 & 0.301 & -0.093 & 0.569 \\
\hline
\end{tabular}

A two-tailed Pearson's partial correlation analysis was performed that controlled for age, sex, educational level, and intracranial volume. The 24 cortical regions that remained significant after Bonferroni correction are listed (corrected $\mathrm{p}=[0.05 / 24]=2.08 \times 10^{-3}$ ). $\mathrm{BD}$, bipolar disorder; HDRS, Hamilton Depression Rating Scale 
Supplementary Table 5. Correlation of YMRS score with cortical surface area in patients with BD

\begin{tabular}{|c|c|c|}
\hline \multirow{2}{*}{ Cortical regions } & \multicolumn{2}{|c|}{ YMRS score } \\
\hline & $\mathrm{R}$ & $\mathrm{p}$ \\
\hline \multicolumn{3}{|l|}{ Left hemisphere } \\
\hline L Pars orbitalis & 0.071 & 0.559 \\
\hline L Long insular gyrus & 0.142 & 0.242 \\
\hline \multicolumn{3}{|l|}{ Right hemisphere } \\
\hline R Frontomarginal gyrus & -0.184 & 0.127 \\
\hline R Subcentral gyrus & 0.077 & 0.525 \\
\hline $\mathrm{R}$ Transverse frontopolar gyrus & -0.123 & 0.312 \\
\hline R Anterior cingulate gyrus & 0.117 & 0.337 \\
\hline R Posterior mid-cingulate gyrus & 0.114 & 0.346 \\
\hline R Ventral posterior cingulate gyrus & -0.097 & 0.423 \\
\hline R Cuneus & 0.066 & 0.588 \\
\hline R Pars opercularis & 0.190 & 0.116 \\
\hline $\mathrm{R}$ Pars orbitalis & -0.072 & 0.551 \\
\hline $\mathrm{R}$ Pars triangularis & -0.105 & 0.388 \\
\hline R Middle frontal gyrus & -0.173 & 0.152 \\
\hline R Superior frontal gyrus & 0.077 & 0.524 \\
\hline $\mathrm{R}$ Long insular gyrus & 0.244 & 0.042 \\
\hline R Short insular gyrus & -0.132 & 0.277 \\
\hline R Superior occipital gyrus & -0.119 & 0.328 \\
\hline R Lingual gyrus & 0.040 & 0.741 \\
\hline R Parahippocampal gyrus & -0.090 & 0.461 \\
\hline R Precuneus & 0.135 & 0.264 \\
\hline R Straight gyrus & 0.097 & 0.423 \\
\hline R Anterior transverse temporal gyrus & -0.085 & 0.484 \\
\hline R Lateral superior temporal gyrus & 0.122 & 0.315 \\
\hline R Planum temporale & -0.112 & 0.355 \\
\hline
\end{tabular}

A two-tailed Pearson's partial correlation analysis was performed that controlled for age, sex, educational level, and intracranial volume. The 24 cortical regions that remained significant after Bonferroni correction are listed (corrected $\mathrm{p}=[0.05 / 24]=2.08 \times 10^{-3}$ ). $\mathrm{BD}$, bipolar disorder; YMRS, Young Mania Rating Scale 
Supplementary Table 6. Correlation of YMRS score with cortical surface areas in patients with BD II

\begin{tabular}{|c|c|c|}
\hline \multirow{2}{*}{ Cortical regions } & \multicolumn{2}{|c|}{ YMRS score } \\
\hline & $\mathrm{R}$ & $\mathrm{p}$ \\
\hline \multicolumn{3}{|l|}{ Left hemisphere } \\
\hline L Pars orbitalis & 0.044 & 0.789 \\
\hline L Long insular gyrus & 0.222 & 0.169 \\
\hline \multicolumn{3}{|l|}{ Right hemisphere } \\
\hline R Frontomarginal gyrus & -0.274 & 0.087 \\
\hline R Subcentral gyrus & 0.318 & 0.046 \\
\hline $\mathrm{R}$ Transverse frontopolar gyrus & -0.259 & 0.107 \\
\hline R Anterior cingulate gyrus & 0.209 & 0.196 \\
\hline R Posterior mid-cingulate gyrus & 0.175 & 0.280 \\
\hline R Ventral posterior cingulate gyrus & -0.206 & 0.203 \\
\hline R Cuneus & 0.255 & 0.113 \\
\hline R Pars opercularis & 0.286 & 0.074 \\
\hline $\mathrm{R}$ Pars orbitalis & -0.202 & 0.211 \\
\hline $\mathrm{R}$ Pars triangularis & -0.228 & 0.156 \\
\hline R Middle frontal gyrus & -0.266 & 0.098 \\
\hline R Superior frontal gyrus & 0.200 & 0.216 \\
\hline R Long insular gyrus & 0.078 & 0.631 \\
\hline R Short insular gyrus & -0.227 & 0.158 \\
\hline R Superior occipital gyrus & -0.084 & 0.605 \\
\hline R Lingual gyrus & 0.132 & 0.418 \\
\hline R Parahippocampal gyrus & -0.161 & 0.320 \\
\hline R Precuneus & 0.258 & 0.107 \\
\hline R Straight gyrus & 0.280 & 0.080 \\
\hline R Anterior transverse temporal gyrus & -0.191 & 0.237 \\
\hline R Lateral superior temporal gyrus & 0.291 & 0.068 \\
\hline R Planum temporale & -0.185 & 0.253 \\
\hline
\end{tabular}

A two-tailed Pearson's partial correlation analysis was performed that controlled for age, sex, educational level, and intracranial volume. The 24 cortical regions that remained significant after Bonferroni correction are listed (corrected $\mathrm{p}=[0.05 / 24]=2.08 \times 10^{-3}$ ). $\mathrm{BD}$, bipolar disorder; YMRS, Young Mania Rating Scale 


\begin{tabular}{|c|c|c|}
\hline \multirow{2}{*}{ Cortical regions } & \multicolumn{2}{|c|}{ All groups } \\
\hline & $\mathrm{F}$ & $\mathrm{p}$ \\
\hline \multicolumn{3}{|l|}{ Left hemisphere } \\
\hline L Frontomarginal gyrus & 0.546 & 0.463 \\
\hline L Inferior occipital gyrus & 0.468 & 0.496 \\
\hline L Paracentral lobule & 0.021 & 0.884 \\
\hline L Subcentral gyrus & 1.442 & 0.234 \\
\hline L Transverse frontopolar gyrus & 0.063 & 0.802 \\
\hline L Anterior cingulate gyrus & 0.346 & 0.559 \\
\hline L Anterior mid-cingulate gyrus & 0.244 & 0.623 \\
\hline L Posterior mid-cingulate gyrus & 0.016 & 0.901 \\
\hline L Dorsal posterior cingulate gyrus & 0.435 & 0.512 \\
\hline L Ventral posterior cingulate gyrus & 2.814 & 0.098 \\
\hline L Cuneus & 0.002 & 0.961 \\
\hline L Pars opercularis & 8.577 & 0.005 \\
\hline L Pars orbitalis & 0.962 & 0.330 \\
\hline L Pars triangularis & 2.497 & 0.119 \\
\hline L Middle frontal gyrus & 2.236 & 0.139 \\
\hline L Superior frontal gyrus & 0.080 & 0.779 \\
\hline L Long insular gyrus & 0.004 & 0.951 \\
\hline L Short insular gyrus & 0.800 & 0.374 \\
\hline L Middle occipital gyrus & 1.157 & 0.286 \\
\hline L Superior occipital gyrus & 0.054 & 0.816 \\
\hline L Lateral occipito-temporal gyrus & 1.283 & 0.261 \\
\hline L Lingual gyrus & 0.569 & 0.453 \\
\hline L Parahippocampal gyrus & 1.072 & 0.304 \\
\hline L Orbital gyrus & 0.245 & 0.622 \\
\hline L Angular gyrus & 4.360 & 0.041 \\
\hline L Supramarginal gyrus & 2.964 & 0.090 \\
\hline L Superior parietal lobule & 1.234 & 0.270 \\
\hline L Postcentral gyrus & 0.039 & 0.845 \\
\hline L Precentral gyrus & 0.789 & 0.378 \\
\hline L Precuneus & 2.120 & 0.150 \\
\hline L Straight gyrus & 0.004 & 0.951 \\
\hline L Subcallosal gyrus & 0.212 & 0.647 \\
\hline L Anterior transverse temporal gyrus & 0.133 & 0.716 \\
\hline L Lateral superior temporal gyrus & 5.289 & 0.025 \\
\hline L Planum polare & 2.149 & 0.147 \\
\hline L Planum temporale & 0.106 & 0.746 \\
\hline L Inferior temporal gyrus & 2.857 & 0.096 \\
\hline L Middle temporal gyrus & 2.191 & 0.143 \\
\hline \multicolumn{3}{|l|}{ Right hemisphere } \\
\hline R Frontomarginal gyrus & 0.022 & 0.884 \\
\hline R Inferior occipital gyrus & 3.120 & 0.082 \\
\hline R Paracentral lobule & 1.286 & 0.261 \\
\hline R Subcentral gyrus & 8.965 & 0.004 \\
\hline $\mathrm{R}$ Transverse frontopolar gyrus & 0.000 & 0.998 \\
\hline $\mathrm{R}$ Anterior cingulate gyrus & 0.000 & 0.997 \\
\hline R Anterior mid-cingulate gyrus & 0.296 & 0.588 \\
\hline $\mathrm{R}$ Posterior mid-cingulate gyrus & 0.430 & 0.514 \\
\hline R Dorsal posterior cingulate gyrus & 2.080 & 0.154 \\
\hline $\mathrm{R}$ Ventral posterior cingulate gyrus & 1.401 & 0.241 \\
\hline $\mathrm{R}$ Cuneus & 0.070 & 0.791 \\
\hline $\mathrm{R}$ Pars opercularis & 10.068 & 0.002 \\
\hline $\mathrm{R}$ Pars orbitalis & 5.762 & 0.019 \\
\hline $\mathrm{R}$ Pars triangularis & 7.248 & 0.009 \\
\hline R Middle frontal gyrus & 2.171 & 0.145 \\
\hline R Superior frontal gyrus & 0.086 & 0.770 \\
\hline R Long insular gyrus & 0.314 & 0.577 \\
\hline R Short insular gyrus & 1.080 & 0.302 \\
\hline R Middle occipital gyrus & 0.835 & 0.364 \\
\hline R Superior occipital gyrus & 0.093 & 0.762 \\
\hline R Lateral occipito-temporal gyrus & 2.199 & 0.143 \\
\hline R Lingual gyrus & 0.115 & 0.736 \\
\hline R Parahippocampal gyrus & 0.016 & 0.900 \\
\hline R Orbital gyrus & 5.042 & 0.028 \\
\hline R Angular gyrus & 4.185 & 0.045 \\
\hline R Supramarginal gyrus & 1.584 & 0.212 \\
\hline R Superior parietal lobule & 1.955 & 0.167 \\
\hline R Postcentral gyrus & 0.203 & 0.654 \\
\hline R Precentral gyrus & 1.391 & 0.242 \\
\hline R Precuneus & 2.637 & 0.109 \\
\hline R Straight gyrus & 0.000 & 1.000 \\
\hline R Subcallosal gyrus & 0.930 & 0.338 \\
\hline $\mathrm{R}$ Anterior transverse temporal gyrus & 0.763 & 0.386 \\
\hline R Lateral superior temporal gyrus & 0.865 & 0.356 \\
\hline R Planum polare & 0.153 & 0.697 \\
\hline R Planum temporale & 4.289 & 0.042 \\
\hline $\mathrm{R}$ Inferior temporal gyrus & 2.281 & 0.136 \\
\hline R Middle temporal gyrus & 4.223 & 0.044 \\
\hline
\end{tabular}


Supplementary Table 8. Effects of lithium on cortical surface area in $\mathrm{BD}$

\begin{tabular}{|c|c|c|}
\hline \multirow{2}{*}{ Cortical regions } & \multicolumn{2}{|c|}{ All groups } \\
\hline & $\mathrm{F}$ & $\mathrm{p}$ \\
\hline \multicolumn{3}{|l|}{ Left hemisphere } \\
\hline L Frontomarginal gyrus & 0.296 & 0.588 \\
\hline L Inferior occipital gyrus & 0.017 & 0.897 \\
\hline L Paracentral lobule & 0.052 & 0.821 \\
\hline L Subcentral gyrus & 0.094 & 0.760 \\
\hline L Transverse frontopolar gyrus & 0.076 & 0.784 \\
\hline L Anterior cingulate gyrus & 1.658 & 0.202 \\
\hline L Anterior mid-cingulate gyrus & 0.050 & 0.823 \\
\hline L Posterior mid-cingulate gyrus & 0.992 & 0.323 \\
\hline L Dorsal posterior cingulate gyrus & 0.523 & 0.472 \\
\hline L Ventral posterior cingulate gyrus & 0.085 & 0.772 \\
\hline LCuneus & 0.436 & 0.511 \\
\hline L Pars opercularis & 2.976 & 0.089 \\
\hline L Pars orbitalis & 0.016 & 0.899 \\
\hline L Pars triangularis & 2.035 & 0.158 \\
\hline L Middle frontal gyrus & 0.216 & 0.643 \\
\hline L Superior frontal gyrus & 1.811 & 0.183 \\
\hline L Long insular gyrus & 0.007 & 0.933 \\
\hline L Short insular gyrus & 0.968 & 0.329 \\
\hline L Middle occipital gyrus & 0.108 & 0.744 \\
\hline L Superior occipital gyrus & 2.152 & 0.147 \\
\hline L Lateral occipito-temporal gyrus & 0.666 & 0.417 \\
\hline L Lingual gyrus & 0.045 & 0.832 \\
\hline L Parahippocampal gyrus & 0.451 & 0.504 \\
\hline L Orbital gyrus & 1.040 & 0.312 \\
\hline L Angular gyrus & 0.198 & 0.657 \\
\hline L Supramarginal gyrus & 0.081 & 0.777 \\
\hline L Superior parietal lobule & 0.531 & 0.469 \\
\hline L Postcentral gyrus & 0.002 & 0.967 \\
\hline L Precentral gyrus & 1.502 & 0.225 \\
\hline L Precuneus & 0.041 & 0.841 \\
\hline L Straight gyrus & 0.007 & 0.933 \\
\hline L Subcallosal gyrus & 0.093 & 0.761 \\
\hline L Anterior transverse temporal gyrus & 1.770 & 0.188 \\
\hline L Lateral superior temporal gyrus & 1.788 & 0.186 \\
\hline L Planum polare & 0.786 & 0.378 \\
\hline L Planum temporale & 0.049 & 0.825 \\
\hline L Inferior temporal gyrus & 0.535 & 0.467 \\
\hline L Middle temporal gyrus & 1.862 & 0.177 \\
\hline \multicolumn{3}{|l|}{ Right hemisphere } \\
\hline R Frontomarginal gyrus & 0.019 & 0.890 \\
\hline $\mathrm{R}$ Inferior occipital gyrus & 0.000 & 0.991 \\
\hline $\mathrm{R}$ Paracentral lobule & 2.195 & 0.143 \\
\hline R Subcentral gyrus & 0.362 & 0.549 \\
\hline $\mathrm{R}$ Transverse frontopolar gyrus & 0.779 & 0.380 \\
\hline $\mathrm{R}$ Anterior cingulate gyrus & 2.256 & 0.138 \\
\hline $\mathrm{R}$ Anterior mid-cingulate gyrus & 0.341 & 0.561 \\
\hline $\mathrm{R}$ Posterior mid-cingulate gyrus & 0.687 & 0.410 \\
\hline $\mathrm{R}$ Dorsal posterior cingulate gyrus & 0.126 & 0.723 \\
\hline$R$ Ventral posterior cingulate gyrus & 0.763 & 0.385 \\
\hline $\mathrm{R}$ Cuneus & 0.034 & 0.855 \\
\hline $\mathrm{R}$ Pars opercularis & 0.047 & 0.829 \\
\hline $\mathrm{R}$ Pars orbitalis & 1.138 & 0.290 \\
\hline $\mathrm{R}$ Pars triangularis & 0.524 & 0.472 \\
\hline $\mathrm{R}$ Middle frontal gyrus & 0.206 & 0.651 \\
\hline R Superior frontal gyrus & 0.235 & 0.629 \\
\hline $\mathrm{R}$ Long insular gyrus & 1.187 & 0.280 \\
\hline R Short insular gyrus & 0.354 & 0.554 \\
\hline R Middle occipital gyrus & 0.015 & 0.904 \\
\hline R Superior occipital gyrus & 1.249 & 0.268 \\
\hline R Lateral occipito-temporal gyrus & 0.005 & 0.945 \\
\hline $\mathrm{R}$ Lingual gyrus & 0.282 & 0.597 \\
\hline R Parahippocampal gyrus & 0.124 & 0.726 \\
\hline R Orbital gyrus & 2.205 & 0.142 \\
\hline R Angular gyrus & 2.220 & 0.141 \\
\hline R Supramarginal gyrus & 0.992 & 0.323 \\
\hline $\mathrm{R}$ Superior parietal lobule & 0.001 & 0.972 \\
\hline $\mathrm{R}$ Postcentral gyrus & 2.270 & 0.137 \\
\hline R Precentral gyrus & 0.003 & 0.955 \\
\hline $\mathrm{R}$ Precuneus & 0.042 & 0.838 \\
\hline R Straight gyrus & 1.601 & 0.210 \\
\hline R Subcallosal gyrus & 0.012 & 0.914 \\
\hline $\mathrm{R}$ Anterior transverse temporal gyrus & 0.948 & 0.334 \\
\hline $\mathrm{R}$ Lateral superior temporal gyrus & 0.135 & 0.714 \\
\hline R Planum polare & 0.269 & 0.606 \\
\hline $\mathrm{R}$ Planum temporale & 0.353 & 0.554 \\
\hline $\mathrm{R}$ Inferior temporal gyrus & 0.122 & 0.728 \\
\hline $\mathrm{R}$ Middle temporal gyrus & 0.227 & 0.635 \\
\hline
\end{tabular}

The general linear model (GLM) included BD patients and adjusted for age, sex, educational level, and intracranial volume as covariates. Regions that remained significant after Bonferroni correction are listed: 76 regions of cortex, corrected $\mathrm{p}=(0.05 / 76)=$ $6.58 \times 10^{-4} . \mathrm{BD}$, bipolar disorder 


\begin{tabular}{|c|c|c|}
\hline \multirow{2}{*}{ Cortical regions } & \multicolumn{2}{|c|}{ All groups } \\
\hline & $\mathrm{F}$ & $\mathrm{p}$ \\
\hline \multicolumn{3}{|l|}{ Left hemisphere } \\
\hline L Frontomarginal gyrus & 3.940 & 0.051 \\
\hline L Inferior occipital gyrus & 0.145 & 0.704 \\
\hline L Paracentral lobule & 0.555 & 0.459 \\
\hline L Subcentral gyrus & 0.063 & 0.802 \\
\hline L Transverse frontopolar gyrus & 0.016 & 0.898 \\
\hline L Anterior cingulate gyrus & 0.503 & 0.481 \\
\hline L Anterior mid-cingulate gyrus & 0.329 & 0.568 \\
\hline L Posterior mid-cingulate gyrus & 0.115 & 0.736 \\
\hline L Dorsal posterior cingulate gyrus & 1.096 & 0.299 \\
\hline L Ventral posterior cingulate gyrus & 0.005 & 0.946 \\
\hline LCuneus & 1.647 & 0.204 \\
\hline L Pars opercularis & 0.123 & 0.727 \\
\hline L Pars orbitalis & 0.052 & 0.820 \\
\hline L Pars triangularis & 0.252 & 0.617 \\
\hline L Middle frontal gyrus & 1.279 & 0.262 \\
\hline L Superior frontal gyrus & 0.195 & 0.660 \\
\hline L Long insular gyrus & 0.885 & 0.350 \\
\hline L Short insular gyrus & 0.566 & 0.454 \\
\hline L Middle occipital gyrus & 0.711 & 0.402 \\
\hline L Superior occipital gyrus & 3.576 & 0.063 \\
\hline L Lateral occipito-temporal gyrus & 0.557 & 0.458 \\
\hline L Lingual gyrus & 1.347 & 0.250 \\
\hline L Parahippocampal gyrus & 0.181 & 0.672 \\
\hline L Orbital gyrus & 0.004 & 0.948 \\
\hline L Angular gyrus & 0.087 & 0.769 \\
\hline L Supramarginal gyrus & 0.017 & 0.898 \\
\hline L Superior parietal lobule & 1.767 & 0.188 \\
\hline L Postcentral gyrus & 0.091 & 0.764 \\
\hline L Precentral gyrus & 1.437 & 0.235 \\
\hline L Precuneus & 1.256 & 0.266 \\
\hline L Straight gyrus & 0.007 & 0.931 \\
\hline L Subcallosal gyrus & 0.021 & 0.886 \\
\hline L Anterior transverse temporal gyrus & 3.237 & 0.076 \\
\hline L Lateral superior temporal gyrus & 2.910 & 0.093 \\
\hline L Planum polare & 0.000 & 0.990 \\
\hline L Planum temporale & 0.257 & 0.614 \\
\hline L Inferior temporal gyrus & 0.394 & 0.532 \\
\hline L Middle temporal gyrus & 0.461 & 0.499 \\
\hline \multicolumn{3}{|l|}{ Right hemisphere } \\
\hline R Frontomarginal gyrus & 0.016 & 0.901 \\
\hline $\mathrm{R}$ Inferior occipital gyrus & 0.597 & 0.442 \\
\hline R Paracentral lobule & 0.424 & 0.517 \\
\hline R Subcentral gyrus & 0.080 & 0.778 \\
\hline $\mathrm{R}$ Transverse frontopolar gyrus & 0.000 & 0.997 \\
\hline $\mathrm{R}$ Anterior cingulate gyrus & 0.176 & 0.676 \\
\hline R Anterior mid-cingulate gyrus & 0.347 & 0.558 \\
\hline $\mathrm{R}$ Posterior mid-cingulate gyrus & 0.004 & 0.951 \\
\hline $\mathrm{R}$ Dorsal posterior cingulate gyrus & 1.252 & 0.267 \\
\hline$R$ Ventral posterior cingulate gyrus & 0.374 & 0.543 \\
\hline $\mathrm{R}$ Cuneus & 1.834 & 0.180 \\
\hline R Pars opercularis & 3.004 & 0.088 \\
\hline R Pars orbitalis & 0.849 & 0.360 \\
\hline $\mathrm{R}$ Pars triangularis & 1.107 & 0.296 \\
\hline $\mathrm{R}$ Middle frontal gyrus & 1.157 & 0.286 \\
\hline R Superior frontal gyrus & 0.018 & 0.894 \\
\hline $\mathrm{R}$ Long insular gyrus & 1.883 & 0.175 \\
\hline R Short insular gyrus & 0.048 & 0.827 \\
\hline R Middle occipital gyrus & 0.134 & 0.715 \\
\hline R Superior occipital gyrus & 1.807 & 0.183 \\
\hline $\mathrm{R}$ Lateral occipito-temporal gyrus & 0.204 & 0.653 \\
\hline $\mathrm{R}$ Lingual gyrus & 1.789 & 0.186 \\
\hline R Parahippocampal gyrus & 0.946 & 0.334 \\
\hline R Orbital gyrus & 0.125 & 0.725 \\
\hline R Angular gyrus & 0.374 & 0.543 \\
\hline R Supramarginal gyrus & 0.184 & 0.669 \\
\hline R Superior parietal lobule & 2.402 & 0.126 \\
\hline R Postcentral gyrus & 0.139 & 0.710 \\
\hline R Precentral gyrus & 0.690 & 0.409 \\
\hline $\mathrm{R}$ Precuneus & 0.433 & 0.513 \\
\hline R Straight gyrus & 1.887 & 0.174 \\
\hline R Subcallosal gyrus & 0.447 & 0.506 \\
\hline $\mathrm{R}$ Anterior transverse temporal gyrus & 1.821 & 0.182 \\
\hline $\mathrm{R}$ Lateral superior temporal gyrus & 0.089 & 0.766 \\
\hline R Planum polare & 0.758 & 0.387 \\
\hline R Planum temporale & 1.811 & 0.183 \\
\hline $\mathrm{R}$ Inferior temporal gyrus & 0.221 & 0.639 \\
\hline R Middle temporal gyrus & 0.363 & 0.549 \\
\hline
\end{tabular}




\begin{tabular}{|c|c|c|}
\hline \multirow{2}{*}{ Cortical regions } & \multicolumn{2}{|c|}{ All groups } \\
\hline & $\mathrm{F}$ & $\mathrm{p}$ \\
\hline \multicolumn{3}{|l|}{ Left hemisphere } \\
\hline L Frontomarginal gyrus & 0.625 & 0.432 \\
\hline L Inferior occipital gyrus & 0.396 & 0.531 \\
\hline L Paracentral lobule & 0.985 & 0.325 \\
\hline L Subcentral gyrus & 2.325 & 0.132 \\
\hline L Transverse frontopolar gyrus & 0.015 & 0.902 \\
\hline L Anterior cingulate gyrus & 0.009 & 0.924 \\
\hline L Anterior mid-cingulate gyrus & 1.779 & 0.187 \\
\hline L Posterior mid-cingulate gyrus & 1.281 & 0.262 \\
\hline L Dorsal posterior cingulate gyrus & 0.682 & 0.412 \\
\hline L Ventral posterior cingulate gyrus & 2.524 & 0.117 \\
\hline LCuneus & 0.558 & 0.458 \\
\hline L Pars opercularis & 0.083 & 0.774 \\
\hline L Pars orbitalis & 0.180 & 0.673 \\
\hline L Pars triangularis & 0.552 & 0.460 \\
\hline L Middle frontal gyrus & 0.936 & 0.337 \\
\hline L Superior frontal gyrus & 0.270 & 0.605 \\
\hline L Long insular gyrus & 1.915 & 0.171 \\
\hline L Short insular gyrus & 2.163 & 0.146 \\
\hline L Middle occipital gyrus & 0.569 & 0.453 \\
\hline L Superior occipital gyrus & 1.418 & 0.238 \\
\hline L Lateral occipito-temporal gyrus & 0.202 & 0.654 \\
\hline L Lingual gyrus & 0.072 & 0.789 \\
\hline L Parahippocampal gyrus & 0.204 & 0.653 \\
\hline L Orbital gyrus & 0.501 & 0.481 \\
\hline L Angular gyrus & 0.011 & 0.916 \\
\hline L Supramarginal gyrus & 0.369 & 0.545 \\
\hline L Superior parietal lobule & 1.826 & 0.181 \\
\hline L Postcentral gyrus & 0.014 & 0.907 \\
\hline L Precentral gyrus & 3.653 & 0.060 \\
\hline L Precuneus & 0.220 & 0.640 \\
\hline L Straight gyrus & 0.236 & 0.628 \\
\hline L Subcallosal gyrus & 0.004 & 0.951 \\
\hline L Anterior transverse temporal gyrus & 0.225 & 0.637 \\
\hline L Lateral superior temporal gyrus & 0.119 & 0.731 \\
\hline L Planum polare & 0.401 & 0.529 \\
\hline L Planum temporale & 0.179 & 0.674 \\
\hline L Inferior temporal gyrus & 0.332 & 0.566 \\
\hline L Middle temporal gyrus & 0.351 & 0.556 \\
\hline \multicolumn{3}{|l|}{ Right hemisphere } \\
\hline R Frontomarginal gyrus & 4.265 & 0.043 \\
\hline $\mathrm{R}$ Inferior occipital gyrus & 0.084 & 0.772 \\
\hline $\mathrm{R}$ Paracentral lobule & 0.051 & 0.822 \\
\hline R Subcentral gyrus & 0.279 & 0.599 \\
\hline $\mathrm{R}$ Transverse frontopolar gyrus & 0.526 & 0.471 \\
\hline $\mathrm{R}$ Anterior cingulate gyrus & 0.876 & 0.352 \\
\hline $\mathrm{R}$ Anterior mid-cingulate gyrus & 1.094 & 0.299 \\
\hline $\mathrm{R}$ Posterior mid-cingulate gyrus & 0.801 & 0.374 \\
\hline $\mathrm{R}$ Dorsal posterior cingulate gyrus & 0.483 & 0.489 \\
\hline$R$ Ventral posterior cingulate gyrus & 0.567 & 0.454 \\
\hline $\mathrm{R}$ Cuneus & 1.059 & 0.307 \\
\hline $\mathrm{R}$ Pars opercularis & 1.894 & 0.173 \\
\hline $\mathrm{R}$ Pars orbitalis & 1.199 & 0.277 \\
\hline $\mathrm{R}$ Pars triangularis & 1.115 & 0.295 \\
\hline $\mathrm{R}$ Middle frontal gyrus & 2.739 & 0.103 \\
\hline R Superior frontal gyrus & 0.432 & 0.513 \\
\hline $\mathrm{R}$ Long insular gyrus & 0.516 & 0.475 \\
\hline R Short insular gyrus & 0.409 & 0.525 \\
\hline R Middle occipital gyrus & 1.159 & 0.285 \\
\hline R Superior occipital gyrus & 3.854 & 0.054 \\
\hline R Lateral occipito-temporal gyrus & 0.557 & 0.458 \\
\hline $\mathrm{R}$ Lingual gyrus & 0.468 & 0.496 \\
\hline R Parahippocampal gyrus & 0.894 & 0.348 \\
\hline R Orbital gyrus & 0.276 & 0.601 \\
\hline R Angular gyrus & 0.157 & 0.693 \\
\hline R Supramarginal gyrus & 0.009 & 0.926 \\
\hline $\mathrm{R}$ Superior parietal lobule & 1.444 & 0.234 \\
\hline $\mathrm{R}$ Postcentral gyrus & 0.222 & 0.639 \\
\hline R Precentral gyrus & 0.979 & 0.326 \\
\hline $\mathrm{R}$ Precuneus & 0.630 & 0.430 \\
\hline R Straight gyrus & 1.737 & 0.192 \\
\hline R Subcallosal gyrus & 0.060 & 0.807 \\
\hline $\mathrm{R}$ Anterior transverse temporal gyrus & 0.770 & 0.383 \\
\hline $\mathrm{R}$ Lateral superior temporal gyrus & 1.434 & 0.235 \\
\hline $\mathrm{R}$ Planum polare & 0.134 & 0.715 \\
\hline $\mathrm{R}$ Planum temporale & 0.957 & 0.331 \\
\hline $\mathrm{R}$ Inferior temporal gyrus & 5.089 & 0.027 \\
\hline $\mathrm{R}$ Middle temporal gyrus & 0.947 & 0.334 \\
\hline
\end{tabular}


Supplementary Table 11. Effects of atypical antipsychotics on cortical thickness in $\mathrm{BD}$

\begin{tabular}{|c|c|c|}
\hline \multirow{2}{*}{ Cortical regions } & \multicolumn{2}{|c|}{ All groups } \\
\hline & $\mathrm{F}$ & $\mathrm{p}$ \\
\hline \multicolumn{3}{|l|}{ Left hemisphere } \\
\hline L Frontomarginal gyrus & 5.016 & 0.028 \\
\hline L Inferior occipital gyrus & 2.662 & 0.107 \\
\hline L Paracentral lobule & 1.028 & 0.314 \\
\hline L Subcentral gyrus & 0.467 & 0.497 \\
\hline L Transverse frontopolar gyrus & 2.044 & 0.157 \\
\hline L Anterior cingulate gyrus & 1.937 & 0.169 \\
\hline L Anterior mid-cingulate gyrus & 2.010 & 0.161 \\
\hline L Posterior mid-cingulate gyrus & 0.075 & 0.785 \\
\hline L Dorsal posterior cingulate gyrus & 2.309 & 0.133 \\
\hline L Ventral posterior cingulate gyrus & 1.495 & 0.226 \\
\hline LCuneus & 0.186 & 0.667 \\
\hline L Pars opercularis & 6.509 & 0.013 \\
\hline L Pars orbitalis & 2.241 & 0.139 \\
\hline L Pars triangularis & 3.777 & 0.056 \\
\hline L Middle frontal gyrus & 2.085 & 0.153 \\
\hline L Superior frontal gyrus & 0.805 & 0.373 \\
\hline L Long insular gyrus & 0.298 & 0.587 \\
\hline L Short insular gyrus & 1.116 & 0.294 \\
\hline L Middle occipital gyrus & 1.473 & 0.229 \\
\hline L Superior occipital gyrus & 0.042 & 0.838 \\
\hline L Lateral occipito-temporal gyrus & 0.613 & 0.437 \\
\hline L Lingual gyrus & 0.133 & 0.717 \\
\hline L Parahippocampal gyrus & 1.710 & 0.195 \\
\hline L Orbital gyrus & 1.515 & 0.223 \\
\hline L Angular gyrus & 3.663 & 0.060 \\
\hline L Supramarginal gyrus & 3.945 & 0.051 \\
\hline L Superior parietal lobule & 0.601 & 0.441 \\
\hline L Postcentral gyrus & 0.010 & 0.921 \\
\hline L Precentral gyrus & 0.947 & 0.334 \\
\hline L Precuneus & 0.225 & 0.637 \\
\hline L Straight gyrus & 0.396 & 0.531 \\
\hline L Subcallosal gyrus & 0.849 & 0.360 \\
\hline L Anterior transverse temporal gyrus & 0.486 & 0.488 \\
\hline L Lateral superior temporal gyrus & 0.662 & 0.419 \\
\hline L Planum polare & 1.976 & 0.164 \\
\hline L Planum temporale & 2.912 & 0.093 \\
\hline L Inferior temporal gyrus & 1.992 & 0.163 \\
\hline L Middle temporal gyrus & 5.027 & 0.028 \\
\hline \multicolumn{3}{|l|}{ Right hemisphere } \\
\hline R Frontomarginal gyrus & 1.090 & 0.300 \\
\hline $\mathrm{R}$ Inferior occipital gyrus & 5.099 & 0.027 \\
\hline $\mathrm{R}$ Paracentral lobule & 2.045 & 0.157 \\
\hline R Subcentral gyrus & 1.217 & 0.274 \\
\hline $\mathrm{R}$ Transverse frontopolar gyrus & 1.080 & 0.302 \\
\hline $\mathrm{R}$ Anterior cingulate gyrus & 0.592 & 0.444 \\
\hline R Anterior mid-cingulate gyrus & 1.112 & 0.295 \\
\hline $\mathrm{R}$ Posterior mid-cingulate gyrus & 0.495 & 0.484 \\
\hline $\mathrm{R}$ Dorsal posterior cingulate gyrus & 0.966 & 0.329 \\
\hline$R$ Ventral posterior cingulate gyrus & 1.717 & 0.195 \\
\hline $\mathrm{R}$ Cuneus & 0.058 & 0.810 \\
\hline $\mathrm{R}$ Pars opercularis & 4.331 & 0.041 \\
\hline $\mathrm{R}$ Pars orbitalis & 1.990 & 0.163 \\
\hline $\mathrm{R}$ Pars triangularis & 15.858 & $1.69 \times 10^{-4}$ \\
\hline $\mathrm{R}$ Middle frontal gyrus & 1.870 & 0.176 \\
\hline R Superior frontal gyrus & 0.461 & 0.499 \\
\hline $\mathrm{R}$ Long insular gyrus & 0.262 & 0.610 \\
\hline R Short insular gyrus & 0.907 & 0.344 \\
\hline R Middle occipital gyrus & 0.814 & 0.370 \\
\hline R Superior occipital gyrus & 0.004 & 0.953 \\
\hline R Lateral occipito-temporal gyrus & 2.303 & 0.134 \\
\hline$R$ Lingual gyrus & 0.140 & 0.710 \\
\hline R Parahippocampal gyrus & 0.297 & 0.587 \\
\hline R Orbital gyrus & 4.073 & 0.048 \\
\hline R Angular gyrus & 2.216 & 0.141 \\
\hline R Supramarginal gyrus & 3.984 & 0.050 \\
\hline R Superior parietal lobule & 3.059 & 0.085 \\
\hline R Postcentral gyrus & 0.394 & 0.532 \\
\hline $\mathrm{R}$ Precentral gyrus & 1.941 & 0.168 \\
\hline $\mathrm{R}$ Precuneus & 0.002 & 0.967 \\
\hline R Straight gyrus & 1.633 & 0.206 \\
\hline R Subcallosal gyrus & 0.017 & 0.896 \\
\hline $\mathrm{R}$ Anterior transverse temporal gyrus & 0.950 & 0.333 \\
\hline $\mathrm{R}$ Lateral superior temporal gyrus & 0.054 & 0.817 \\
\hline $\mathrm{R}$ Planum polare & 1.235 & 0.270 \\
\hline R Planum temporale & 0.000 & 0.999 \\
\hline $\mathrm{R}$ Inferior temporal gyrus & 2.130 & 0.149 \\
\hline R Middle temporal gyrus & 3.561 & 0.063 \\
\hline
\end{tabular}

The general linear model (GLM) included BD patients and adjusted for age, sex, educational level, and intracranial volume as covariates. Regions that remained significant after Bonferroni correction are listed: 76 regions of cortex, corrected $\mathrm{p}=(0.05 / 76)=$ $6.58 \times 10^{-4} . \mathrm{BD}$, bipolar disorder 


\begin{tabular}{|c|c|c|}
\hline \multirow{2}{*}{ Cortical regions } & \multicolumn{2}{|c|}{ All groups } \\
\hline & $\mathrm{F}$ & $\mathrm{p}$ \\
\hline \multicolumn{3}{|l|}{ Left hemisphere } \\
\hline L Frontomarginal gyrus & 2.308 & 0.133 \\
\hline L Inferior occipital gyrus & 1.672 & 0.200 \\
\hline L Paracentral lobule & 0.098 & 0.755 \\
\hline L Subcentral gyrus & 0.464 & 0.498 \\
\hline L Transverse frontopolar gyrus & 2.080 & 0.154 \\
\hline L Anterior cingulate gyrus & 3.521 & 0.065 \\
\hline L Anterior mid-cingulate gyrus & 0.032 & 0.859 \\
\hline L Posterior mid-cingulate gyrus & 0.045 & 0.833 \\
\hline L Dorsal posterior cingulate gyrus & 0.163 & 0.688 \\
\hline L Ventral posterior cingulate gyrus & 1.441 & 0.234 \\
\hline LCuneus & 0.008 & 0.930 \\
\hline L Pars opercularis & 0.093 & 0.762 \\
\hline L Pars orbitalis & 0.970 & 0.328 \\
\hline L Pars triangularis & 0.028 & 0.868 \\
\hline L Middle frontal gyrus & 0.490 & 0.486 \\
\hline L Superior frontal gyrus & 1.018 & 0.317 \\
\hline L Long insular gyrus & 1.420 & 0.237 \\
\hline L Short insular gyrus & 0.248 & 0.620 \\
\hline L Middle occipital gyrus & 1.765 & 0.188 \\
\hline L Superior occipital gyrus & 0.000 & 0.983 \\
\hline L Lateral occipito-temporal gyrus & 0.046 & 0.831 \\
\hline L Lingual gyrus & 0.160 & 0.691 \\
\hline L Parahippocampal gyrus & 0.214 & 0.645 \\
\hline L Orbital gyrus & 0.996 & 0.322 \\
\hline L Angular gyrus & 0.002 & 0.963 \\
\hline L Supramarginal gyrus & 5.487 & 0.022 \\
\hline L Superior parietal lobule & 3.602 & 0.062 \\
\hline L Postcentral gyrus & 0.109 & 0.742 \\
\hline L Precentral gyrus & 0.376 & 0.542 \\
\hline L Precuneus & 4.706 & 0.034 \\
\hline L Straight gyrus & 0.097 & 0.756 \\
\hline L Subcallosal gyrus & 0.222 & 0.639 \\
\hline L Anterior transverse temporal gyrus & 0.326 & 0.570 \\
\hline L Lateral superior temporal gyrus & 2.345 & 0.130 \\
\hline L Planum polare & 1.554 & 0.217 \\
\hline L Planum temporale & 0.030 & 0.862 \\
\hline L Inferior temporal gyrus & 2.856 & 0.096 \\
\hline L Middle temporal gyrus & 0.798 & 0.375 \\
\hline \multicolumn{3}{|l|}{ Right hemisphere } \\
\hline R Frontomarginal gyrus & 2.455 & 0.122 \\
\hline $\mathrm{R}$ Inferior occipital gyrus & 0.164 & 0.687 \\
\hline $\mathrm{R}$ Paracentral lobule & 0.002 & 0.969 \\
\hline R Subcentral gyrus & 0.297 & 0.587 \\
\hline $\mathrm{R}$ Transverse frontopolar gyrus & 0.524 & 0.471 \\
\hline $\mathrm{R}$ Anterior cingulate gyrus & 0.491 & 0.486 \\
\hline $\mathrm{R}$ Anterior mid-cingulate gyrus & 0.006 & 0.937 \\
\hline $\mathrm{R}$ Posterior mid-cingulate gyrus & 0.284 & 0.596 \\
\hline $\mathrm{R}$ Dorsal posterior cingulate gyrus & 0.065 & 0.799 \\
\hline$R$ Ventral posterior cingulate gyrus & 0.779 & 0.381 \\
\hline $\mathrm{R}$ Cuneus & 2.310 & 0.133 \\
\hline $\mathrm{R}$ Pars opercularis & 0.620 & 0.434 \\
\hline $\mathrm{R}$ Pars orbitalis & 0.453 & 0.503 \\
\hline $\mathrm{R}$ Pars triangularis & 0.896 & 0.347 \\
\hline $\mathrm{R}$ Middle frontal gyrus & 1.577 & 0.214 \\
\hline R Superior frontal gyrus & 0.733 & 0.395 \\
\hline $\mathrm{R}$ Long insular gyrus & 2.905 & 0.093 \\
\hline R Short insular gyrus & 1.214 & 0.274 \\
\hline R Middle occipital gyrus & 0.012 & 0.914 \\
\hline R Superior occipital gyrus & 0.954 & 0.332 \\
\hline R Lateral occipito-temporal gyrus & 2.112 & 0.151 \\
\hline $\mathrm{R}$ Lingual gyrus & 1.259 & 0.266 \\
\hline R Parahippocampal gyrus & 0.736 & 0.394 \\
\hline R Orbital gyrus & 1.144 & 0.289 \\
\hline R Angular gyrus & 0.002 & 0.963 \\
\hline R Supramarginal gyrus & 0.583 & 0.448 \\
\hline $\mathrm{R}$ Superior parietal lobule & 0.175 & 0.677 \\
\hline $\mathrm{R}$ Postcentral gyrus & 0.874 & 0.353 \\
\hline R Precentral gyrus & 0.019 & 0.890 \\
\hline $\mathrm{R}$ Precuneus & 0.169 & 0.682 \\
\hline R Straight gyrus & 0.490 & 0.486 \\
\hline R Subcallosal gyrus & 0.068 & 0.795 \\
\hline $\mathrm{R}$ Anterior transverse temporal gyrus & 0.555 & 0.459 \\
\hline $\mathrm{R}$ Lateral superior temporal gyrus & 0.247 & 0.621 \\
\hline R Planum polare & 3.056 & 0.085 \\
\hline $\mathrm{R}$ Planum temporale & 0.572 & 0.452 \\
\hline $\mathrm{R}$ Inferior temporal gyrus & 1.715 & 0.195 \\
\hline $\mathrm{R}$ Middle temporal gyrus & 0.050 & 0.825 \\
\hline
\end{tabular}

